\title{
On the local meromorphic extension of CR meromorphic mappings
}

\author{
by JoËL Merker (Marseille) and Egmont Porten (Berlin)
}

\begin{abstract}
Let $M$ be a generic CR submanifold in $\mathbb{C}^{m+n}, m=\operatorname{CRdim} M \geq 1$, $n=\operatorname{codim} M \geq 1, d=\operatorname{dim} M=2 m+n$. A CR meromorphic mapping (in the sense of Harvey-Lawson) is a triple $\left(f, \mathcal{D}_{f},\left[\Gamma_{f}\right]\right)$, where: 1$) f: \mathcal{D}_{f} \rightarrow Y$ is a $\mathcal{C}^{1}$-smooth mapping defined over a dense open subset $\mathcal{D}_{f}$ of $M$ with values in a projective manifold $Y$; 2) the closure $\Gamma_{f}$ of its graph in $\mathbb{C}^{m+n} \times Y$ defines an oriented scarred $\mathcal{C}^{1}$-smooth CR manifold of CR dimension $m$ (i.e. CR outside a closed thin set) and 3) $d\left[\Gamma_{f}\right]=0$ in the sense of currents. We prove that $\left(f, \mathcal{D}_{f},\left[\Gamma_{f}\right]\right)$ extends meromorphically to a wedge attached to $M$ if $M$ is everywhere minimal and $\mathcal{C}^{\omega}$ (real-analytic) or if $M$ is a $\mathcal{C}^{2, \alpha}$ globally minimal hypersurface.
\end{abstract}

Since the works of Trépreau, Tumanov and Jöricke, extendability properties of $\mathrm{CR}$ functions on a smooth $\mathrm{CR}$ manifold $M$ became fairly well understood. In a natural way, 1) $M$ is seen to be a disjoint union of CR bricks, called $C R$ orbits, each of which being an immersed CR submanifold of $M$ with the same CR dimension as $M([21]) ; 2)$ a continuous CR function $f$ on $M$ is CR if and only if its restriction $\left.f\right|_{\mathcal{O}_{C R}}$ is CR on each CR orbit $\left.\mathcal{O}_{C R}([9],[17],[16]) ; 3\right)$ for each $\mathrm{CR}$ orbit $\mathcal{O}_{C R}$, there exists an analytic wedge $\mathcal{W}^{\text {an }}$ attached to $\mathcal{O}_{C R}$, i.e. a conic complex manifold with edge $\mathcal{O}_{C R}$ and with $\operatorname{dim}_{\mathbb{C}} \mathcal{W}^{\text {an }}=\operatorname{dim}_{\mathbb{R}}\left(\mathcal{O}_{C R}\right)-\operatorname{CRdim} M$, such that each continuous CR function on $\mathcal{O}_{C R}$ admits a holomorphic extension to $\mathcal{W}^{\text {an }}$ ([21], [9], [22], [14]). The technique of FBI transforms ([21]) or deformations of analytic discs ([22], [8], [14]) brings up the construction of the analytic wedges in a semi-local way.

This paper is devoted to the question of meromorphic extension to wedges of CR meromorphic functions in the sense of Harvey and Lawson ([5], see also [19]).

1991 Mathematics Subject Classification: Primary 32D20, 32A20, 32D10, 32C16; Secondary $32 \mathrm{~F} 40$.

Key words and phrases: CR generic currents, scarred CR manifolds, removable singularities for CR functions, deformations of analytic discs, CR meromorphic mappings. 
The classical theorem of Hartogs-Levi states that, if a meromorphic function is given on a neighborhood $\mathcal{V}(b \Omega)$ of the boundary of a bounded domain $\Omega \Subset \mathbb{C}^{m+1}, m+1 \geq 2$, then it extends meromorphically inside $\Omega$. Using the solution of the complex Plateau problem, i.e. attaching holomorphic chains to maximally complex cycles in the complex euclidean space, Harvey and Lawson proved the following Hartogs-Bochner theorem for meromorphic maps: If $m+1 \geq 3$, then any CR mapping $b \Omega \rightarrow Y$, with values in a projective manifold $Y$, extends meromorphically to $\Omega$. The method allows indeterminacies: a CR meromorphic mapping is defined by Harvey and Lawson as a triple $\left(f, \mathcal{D}_{f},\left[\Gamma_{f}\right]\right)$, where $f: \mathcal{D}_{f} \rightarrow Y$ is a $\mathcal{C}^{1}$-smooth mapping defined over a dense open subset $\mathcal{D}_{f} \subset M=b \Omega$ with values in a projective manifold $Y$; the closure $\Gamma_{f}$ of its graph in $\mathbb{C}^{m+1} \times Y$ defines a scarred $\mathcal{C}^{1}$-smooth $\mathrm{CR}$ manifold of $\mathrm{CR}$ dimension $m$ (i.e. $\mathrm{CR}$ outside a closed thin set) and such that $d\left[\Gamma_{f}\right]=0$ in the sense of currents. The case $m=1$ was open until Dolbeault and Henkin gave a positive answer for $\mathcal{C}^{2}$ CR mappings $f$ using their solution of the boundary problem in $\mathbb{P}^{n}(\mathbb{C}), n \geq 2([4])$. For continuous $f$ with values in a compact Kähler manifold the second-named author devised a different proof, relying on the fact that $b \Omega$ is a single CR orbit and that the envelope of holomorphy of $\mathcal{V}(b \Omega)$ contains $\Omega$ ([18], see Section 4).

Recently, Sarkis obtained the analog of the Hartogs-Bochner theorem for meromorphic maps, allowing indeterminacies ([19], see Section 4). The main idea is to see that the set $\Sigma_{f}$ of indeterminacies of $\left(f, \mathcal{D}_{f},\left[\Gamma_{f}\right]\right)$ is a closed subset with empty interior of some $\mathcal{C}^{1}$-scarred submanifold $\Lambda \subset M=b \Omega$, with $\operatorname{codim}_{M} \Lambda=2$, and that $f$ defines an order zero CR distribution on $M \backslash \Sigma_{f}$. Then the question of CR meromorphic extension is reduced to the local removable singularities theorems in the spirit of Jöricke ([7], [8], [9]). We would like to mention that these removability results were originally impulsed by Jöricke in [7] and in [9].

The goal of this article is to push forward meromorphic extension on CR manifolds of arbitrary codimension, the analogs of domains being wedges over CR manifolds. It seems natural to use the theory of Trépreau-Tumanov in this context. Knowing thinness of $\Sigma_{f}$ (Sarkis) and using wedge removable singularities theorems $([15],[16],[17])$, we prove in this paper that a CR meromorphic mapping $\left(f, \mathcal{D}_{f},\left[\Gamma_{f}\right]\right)$ extends meromorphically to a wedge attached to $M$ if the CR generic manifold $M$ is everywhere minimal in the sense of Tumanov and real-analytic, $\mathcal{C}^{\omega}$-smooth. We also prove that such $\mathrm{CR}$ meromorphic mappings extend meromorphically to a wedge if $M$ is a $\mathcal{C}^{2, \alpha_{-}}$ smooth $(0<\alpha<1)$ hypersurface in $\mathbb{C}^{m+1}$ that is only globally minimal and we prove the meromorphic extension in any codimension if $M$ is everywhere minimal and if the scar set $\operatorname{Sc}\left(\Sigma_{f}\right)$ (in fact $\operatorname{Sc}(\Lambda)$ ) of the indeterminacy set $\Sigma_{f}$ is of $(d-3)$-dimensional Hausdorff measure zero, $d=2 m+n=\operatorname{dim} M$. These results are parallel to the meromorphic extension theorem obtained 
by Dinh and Sarkis for manifolds $M$ with nondegenerate vector-valued Leviform $([3])$.

We refer the reader to Section 4 which plays the role of a detailed introduction.

Acknowledgements. We are grateful to Professor Henkin who raised the question. We also wish to address special thanks to Frederic Sarkis. He has communicated to us the reduction of meromorphic extension of CR meromorphic mappings to a removable singularity property and we had several interesting conversations with him.

1. Currents and scarred manifolds. In this section, we follow Harvey and Lawson for a preliminary exposition of currents in the CR category. This material is known, and is recalled here for clarity. Let $\mathcal{U} \subset \mathbb{C}^{m+n}$ be an open set. We denote by $\mathcal{D}^{k}(\mathcal{U})$ the space of all complex-valued $\mathcal{C}^{\infty}$ exterior $k$-forms on $\mathcal{U}$ with the usual topology. The dual space to $\mathcal{D}^{k}(\mathcal{U})$ will be denoted by $\mathcal{D}_{k}^{\prime}(\mathcal{U})$. We adopt the dual notation $\mathcal{D}_{k}^{\prime}(\mathcal{U})=\mathcal{D}^{\prime 2(m+n)-k}(\mathcal{U})$ and say that elements of this space are currents of dimension $k$ and degree $2(m+n)-k$ on $\mathcal{U}$. In fact, every $k$-dimensional current can be naturally represented as an exterior $(2(m+n)-k)$-form on $\mathcal{U}$ with coefficients in $\mathcal{D}_{2 m+2 n}^{\prime}(\mathcal{U})$.

We let $d: \mathcal{D}^{k}(\mathcal{U}) \rightarrow \mathcal{D}^{k+1}(\mathcal{U})$ denote the exterior differentiation operator and also denote by $d: \mathcal{D}_{k+1}^{\prime}(\mathcal{U}) \rightarrow \mathcal{D}_{k}^{\prime}(\mathcal{U})$ the adjoint map (i.e. $\left.d: \mathcal{D}^{\prime 2(m+n)-k-1}(\mathcal{U}) \rightarrow \mathcal{D}^{\prime 2(m+n)-k}(\mathcal{U})\right)$.

In the following, $\mathcal{H}^{q}, q \in \mathbb{R}, 0 \leq q \leq 2 m+2 n$, will denote the Hausdorff $q$-dimensional measure on $\mathbb{C}^{m+n}$. The notation $\mathcal{H}_{\mathrm{loc}}^{q}(E)<\infty$ for a set $E \subset$ $\mathbb{C}^{m+n}$ means that, for all compact subsets $K \Subset E, \mathcal{H}^{q}(K)<\infty$ (we refer the reader to the paragraph before Proposition 5.7 for a presentation of Hausdorff measures).

We have the Dolbeault decomposition $\mathcal{D}^{k}(\mathcal{U})=\bigoplus_{r+s=k} \mathcal{D}^{r, s}(\mathcal{U})$ and its dual decomposition $\mathcal{D}_{k}^{\prime}(\mathcal{U})=\bigoplus_{r+s=k} \mathcal{D}_{r, s}^{\prime}(\mathcal{U})$ (or $\mathcal{D}^{\prime 2(m+n)-k}(\mathcal{U})=$ $\left.\bigoplus_{r+s=k} \mathcal{D}^{\prime m+n-r, m+n-s}(\mathcal{U})\right)$. A current in $\mathcal{D}_{r, s}^{\prime}(\mathcal{U})=\mathcal{D}^{\prime m+n-r, m+n-s}(\mathcal{U})$ is said to have bidimension $(r, s)$ and bidegree $(m+n-r, m+n-s)$. Given a current $T \in \mathcal{D}_{k}^{\prime}(\mathcal{U})$, we denote the components of $T$ in the space $\mathcal{D}_{r, s}^{\prime}(\mathcal{U})=\mathcal{D}^{\prime m+n-r, m+n-s}(\mathcal{U})$ by $T_{r, s}$ or $T^{m+n-r, m+n-s}$ : the subscripts refer to bidimension and the superscripts to bidegree. Thus

$$
\mathcal{D}_{k}^{\prime}(\mathcal{U}) \ni T=\sum_{r+s=k} T_{r, s}=\sum_{r+s=k} T^{m+n-r, m+n-s} .
$$

Let $M$ be an oriented $d$-dimensional manifold of class $\mathcal{C}^{1}$ in $\mathcal{U}$ with $\mathcal{H}_{\text {loc }}^{d}(M)<\infty$. Then $M$ defines a current $[M] \in \mathcal{D}_{d}^{\prime}(\mathcal{U})$, called the current of integration on $M$, by $[M](\varphi)=\int_{M} \varphi$ for all $\varphi \in \mathcal{D}^{q}(\mathcal{U})$. Furthermore, $d[M]=0$ if $b M=\emptyset$ by Stokes' formula, in particular if $M$ is a closed 
submanifold of $\mathcal{U}$. An obvious remark is that $[M]=[M \backslash \sigma]$ for all closed sets $\sigma \subset \mathcal{U}$ with $\mathcal{H}^{d}(\sigma)=0$. For example, pure $d$-dimensional real or complex analytic sets $\Psi \subset \mathcal{U}$ have a geometric decomposition into a regular and a singular part, $\Psi=\operatorname{Reg}(\Psi) \cup \operatorname{Sing}(\Psi)$, with $\operatorname{Reg}(\Psi) \cap \operatorname{Sing}(\Psi)=\emptyset \cdot \operatorname{Reg}(\Psi)$ is a closed $d$-dimensional submanifold of $M \backslash \operatorname{Sing}(\Psi)$ and $\mathcal{H}^{d}(\operatorname{Sing}(\Psi))=0$, so one can define $[\Psi]=[\Psi \backslash \operatorname{Sing}(\Psi)]=[\operatorname{Reg}(\Psi)]$. In the smooth category, it is convenient to set up the following definition. Let $r \geq 1$ and work in the $\mathcal{C}^{r}$ category, $r \geq 1$.

1.1. Definition ([5], [19]). A closed set $M$ in a real manifold $X$ is called a $\mathcal{C}^{r}$-scarred manifold of dimension $d$ if there exists a closed set $\sigma \subset M$ with $\mathcal{H}_{\mathrm{loc}}^{d}(\sigma)=0$ such that $M \backslash \sigma$ is an oriented $\mathcal{C}^{r}$-smooth $d$-dimensional submanifold of $X \backslash \sigma$ with $\mathcal{H}_{\text {loc }}^{d}(M \backslash \sigma)<\infty$.

The smallest set $\sigma \subset M$ with the above properties is called the scarred set of $M$. We adopt the notation $\sigma=\operatorname{Sc}(M)$ and $\operatorname{Reg}(M)=M \backslash \operatorname{Sc}(M)$. Nonetheless, if $M$ is $\mathcal{C}^{r}$-smooth, then $d[M]=0$ of course does not imply that $d[M \backslash \sigma]=0$ for a set $\sigma \subset M$ with $\mathcal{H}_{\text {loc }}^{d}(\sigma)=0$.

Let $M$ be a $\mathcal{C}^{r}$-scarred manifold of dimension $d$. It follows from Stokes' formula that, if $\mathcal{H}_{\mathrm{loc}}^{d-1}(\mathrm{Sc}(M))=0$, then the current $[M]$ has no boundary, i.e. $d[M]=0$, in particular if $M$ is a complex-analytic set. The current $[M]$ given by integration over $M \backslash \operatorname{Sc}(M)$ is well defined, but to retain the local behavior of a smooth current of integration, one must add the condition that $d[M]=0$ locally, or globally, to have a globally closed object, for example to solve a boundary problem.

When $M$ is noncompact, the condition $d[M]=0$ will mean the following: $d([M] \cap U)=0$ for each open set $U \Subset X$ with $\operatorname{Int} \bar{U}=U$. One says that $d[M]=0$ locally.

1.2. Definition. $M$ is called a $\mathcal{C}^{r}$-scarred cycle if, moreover, $d[M]=0$ locally.

This condition is geometric in nature and is rather independent of the measure-theoretic largeness expressed by $\mathcal{H}_{\text {loc }}^{d}(\operatorname{Sc}(M))=0$. It corrects the singularities globally (think of $\operatorname{dim} M=1$ ).

2. Geometry of $M$ and CR currents. Our purpose in this section is to study the meaning of the notion of a CR meromorphic mapping $\left(f, \mathcal{D}_{f},\left[\Gamma_{f}\right]\right)$ in the sense of Harvey and Lawson, in particular the implications of the fact that $\left[\Gamma_{f}\right]$ defines a $\mathcal{C}^{r}$-scarred manifold. Following [5], we begin by establishing various useful equivalent formulations of the notion of CR functions. These definitions take place in the category of CR objects and CR manifolds. Any locally embeddable CR manifold being embeddable as a piece of a generic submanifold in $\mathbb{C}^{m+n}$, i.e. with $\mathrm{CRdim} M=m$ and $\operatorname{codim} M=n$, we set up these concepts for $M$ generic. 
Let $M$ be a $\mathcal{C}^{r}$-scarred CR manifold of type $(m, n)$ in $\mathbb{C}^{m+n}$, i.e. of dimension $2 m+n$, of $\mathrm{CR}$ dimension $m$ and of codimension $n$. Denote by $t \in \mathbb{C}^{m+n}$ the coordinates on $\mathbb{C}^{m+n}$. Near a point $p_{0} \in \operatorname{Reg}(M), M$ can be defined by cartesian equations $\varrho_{j}(t)=0,1 \leq j \leq n$, where $\partial \varrho_{1} \wedge \ldots \wedge \partial \varrho_{n}$ does not vanish on $M$. We then have

$$
T_{p}^{c} M=T_{p} M \cap J T_{p} M=\left\{X \in T_{p} M: \partial \varrho_{j}(X)=0, j=1, \ldots, n\right\},
$$

where $J$ denotes the usual complex structure on $T \mathbb{C}^{m+n}$. Then $J$ can be extended to the complexification $T_{p}^{c} M \otimes_{\mathbb{R}} \mathbb{C}$ with eigenvalues $\pm i$. Let $T_{p}^{c} M \otimes$ $\mathbb{C}=T^{1,0} M \oplus T^{0,1} M$ denote the decomposition into the eigenspaces for $i$ and $-i$ respectively. Then there is a natural $\mathbb{C}$-linear isomorphism from $T_{p}^{c} M$ to $T_{p}^{1,0} M$ given by the correspondence $X \mapsto Z=\frac{1}{2}(X-i J X)$. Moreover, the operation of complex conjugation is well-defined on $T_{p}^{c} M \otimes_{\mathbb{R}} \mathbb{C}$ and we have $T_{p}^{1,0} M=\overline{T_{p}^{0,1} M}$.

Suppose now that $f: M \rightarrow \mathbb{C}$ is a function of class $\mathcal{C}^{1} . f$ is called a $C R$ function if $\bar{L} f=0$, for every section $\bar{L}$ of $T^{0,1} M$, i.e. $f$ is annihilated by the antiholomorphic vectors tangent to $M$. Equivalently, the differential $d f$ is complex-linear at each point $p \in M, d f(J X)=i d f(X)$ for all $X \in T_{p}^{c} M$. The first definition still makes sense for the wider class of CR distributions on $M$.

To check a generalized definition in the distributional sense, let $U \subset$ $M$ be a small open set, let $l_{1}, \ldots, l_{m} \in \Gamma\left(U, T^{c} M\right)$ and let $\lambda_{1}, \ldots, \lambda_{n} \in$ $\Gamma(U, T M)$ with $l_{1}, J l_{1}, \ldots, l_{m}, J l_{m}, \lambda_{1}, \ldots, \lambda_{n}$ linearly independent.

These vector fields determine splittings $T U=T^{c} U \oplus \Lambda_{U}$ and $T^{*} U=$ $T^{c} U^{*} \oplus \Lambda_{U}^{*}$ of the tangent bundle and the cotangent bundle $T^{*} M$ restricted to $U$. The two spaces $T^{c} M$, called the complex tangent bundle, and $H^{0} M=$ $\left(T^{c} M\right)^{\perp}$, the annihilator of $T^{c} M$ in $T^{*} M$, called the characteristic bundle of $M$, are canonical; the other two depend on the choice of a splitting. Let $l_{1}^{*}, J l_{1}^{*}, \ldots, l_{m}^{*}, J l_{m}^{*}, \lambda_{1}^{*}, \ldots, \lambda_{n}^{*}$ be the dual covector fields. Naturally, if $f \in \mathcal{C}^{1}(U, \mathbb{C})$ then

$$
d f=\sum_{j=1}^{m}\left(l_{j}(f) l_{j}^{*}+J l_{j}(f) J l_{j}^{*}\right)+\sum_{k=1}^{n} \lambda_{k}(f) \lambda_{k}^{*} .
$$

Then one can define an induced $\bar{\partial}$ operator on $M$ by

$$
\bar{\partial}_{M}(f)=\sum_{j=1}^{m} \bar{L}_{j}(f) \bar{L}_{j}^{*},
$$

where $\bar{L}_{j}=\frac{1}{2}\left(l_{j}+i J l_{j}\right)$ and $\bar{L}_{j}^{*}=\left(l_{j}^{*}-i J l_{j}^{*}\right)$ for $j=1, \ldots, m$. Clearly, the kernel of $\bar{\partial}_{M}$ is the ring $\mathrm{CR}(M)$ of CR functions on $M$, and the definition of $\bar{\partial}_{M}$ is independent of the choice of local vector fields. However, the operator does depend on the choice of the splitting of $T M$. 
Note that if we extend the local vector fields used in the definition of $\bar{\partial}_{M}$ above to a neighborhood $\mathcal{U}$ of $U$ in $\mathbb{C}^{m+n}$, then we have

$$
\bar{\partial}(f)=\sum_{j=1}^{m} \bar{L}_{j}(f) \bar{L}_{j}^{*}+\sum_{k=1}^{n} \bar{\Lambda}_{k}(f) \bar{\Lambda}_{k}^{*}
$$

where $\bar{\Lambda}_{k}=\frac{1}{2}\left(\lambda_{k}+i J \lambda_{k}\right)$ and $\bar{\Lambda}_{k}^{*}=\left(\lambda_{k}^{*}-i J \lambda_{k}^{*}\right)$ for $k=1, \ldots, n$. If, furthermore, $M=\left\{\varrho_{1}=\ldots=\varrho_{n}=0\right\}$ as above, then along $M$ we can assume that $\lambda_{k}^{*}=\partial \varrho_{k}=d \varrho_{k}+i d^{c} \varrho_{k}$.

2.1. Proposition. Let $M$ be a piece of a $\mathcal{C}^{1}$-smooth manifold with $\operatorname{dim} M$ $=2 m+n$, closed in an open set $\mathcal{U} \subset \mathbb{C}^{m+n}$. Then the following conditions are equivalent.

(i) $\operatorname{dim}_{\mathbb{C}} T_{p} M \cap J T_{p} M=m$ for all $p \in M$;

(ii) $\int_{M} \alpha=0$ for all $(r, s)$-forms $\alpha$ on $\mathcal{U}$ with $r+s=2 m+n$ and $|r-s|>n$;

(iii) $[M]=[M]_{m, m+n}+[M]_{m+1, m+n-1}+\ldots+[M]_{m+n, m}$, where $[M]_{r, s}$ are the components of the current of integration $[M]$ with respect to the Dolbeault decomposition;

(iv) $M$ is locally given by $n$ scalar equations $x_{j}=h_{j}(y, w), j=1, \ldots, n$, in holomorphic coordinates $t=(w, z), w \in \mathbb{C}^{m}, z=x+i y \in \mathbb{C}^{n}$, with $h_{j}(0)=0$ and $d h_{j}(0)=0$.

The proof is omitted. When $M$ is $\mathcal{C}^{1}$-scarred, it is natural to allow singularities also for maps defined over $M$. The precise formulation is due to Harvey and Lawson ([5], II) and favors the graph viewpoint. We transpose it in the CR category.

2.2. Definition ([5]). Let $M$ be a $\mathcal{C}^{r}$-scarred submanifold of $\mathbb{C}^{m+n}$. Then a $\mathcal{C}^{r}$-scarred mapping of $M$ into a complex manifold $Y$ is a $\mathcal{C}^{r}$-smooth map $f: \mathcal{D}_{f} \rightarrow Y$ defined on an open dense subset $\mathcal{D}_{f} \subset \operatorname{Reg}(M)$ such that the closure $\Gamma_{f}$ of the graph $\left\{(p, f(p)) \in \mathcal{D}_{f} \times Y\right\}$ in $\mathbb{C}^{m+n} \times Y$ defines a $\mathcal{C}^{r}$-scarred cycle in $\mathbb{C}^{m+n} \times Y$, i.e. $d\left[\Gamma_{f}\right]=0$.

$\mathcal{C}^{r}$-scarred mappings will constantly be denoted by $\left(f, \mathcal{D}_{f},\left[\Gamma_{f}\right]\right)$, to remind precisely that they are not set-theoretic maps.

2.3. Definition ([5], [19]). $\left(f, \mathcal{D}_{f},\left[\Gamma_{f}\right]\right)$ is called a $\mathcal{C}^{r}$-scarred $C R$ mapping if, moreover, $\left[\Gamma_{f}\right]$ is a $\mathcal{C}^{r}$-scarred CR cycle of $\mathbb{C}^{m+n} \times Y$ of CR dimension $m$.

One can go a step further in generalization. Indeed, Harvey and Lawson have introduced the notion of maximally complex currents. Accordingly, CR currents arise as generalized currents of integration on CR manifolds as follows. 
2.4. Definition. Let $\mathcal{M}$ be a $d=(2 m+n)$-dimensional current with compact support on an $(m+n)$-dimensional complex manifold $X . \mathcal{M}$ is called a generic current of type $(m, n)$ if its Dolbeault components satisfy

$$
\mathcal{M}_{r, s}=0, \quad r+s=2 m+n, \text { for }|r-s|>n,
$$

i.e. $\mathcal{M}(\alpha)=0$ for all $(r, s)$-forms $\alpha$ on $X$ with $|r-s|>n$.

Let $\mathcal{M}$ be a closed generic current of type $(m, n)$ in an open set $\mathcal{U} \subset$ $\mathbb{C}^{m+n}$. Then $\mathcal{M}=\mathcal{M}^{0, n}+\ldots+\mathcal{M}^{n, 0}$ and $d \mathcal{M}=0$ yield $\bar{\partial} \mathcal{M}^{0, n}=0$, since $[d \mathcal{M}]^{0, n+1}=\bar{\partial} \mathcal{M}^{0, n}$, simply for bidegree reasons. Using this remark yields four equivalent definitions for a $\mathcal{C}^{1}$-smooth function to be CR. $\left[\Gamma_{f}\right]$ denotes the current of integration over the closure of the graph of $f$. Since $d\left[\Gamma_{f}\right]=0, \bar{\partial}\left[\Gamma_{f}\right]^{0, n}=0$. The variable $\zeta$ is used to denote a coordinate on $\mathbb{C}$, and $f: M \rightarrow \mathbb{C}$. Property (iv) below can be used as a new definition. Let $\pi$ denote the projection $\mathbb{C}^{m+n} \times \mathbb{C} \rightarrow \mathbb{C}^{m+n}$.

2.5. Proposition ([5]). Let $M$ be an oriented real $C R$ manifold of class $\mathcal{C}^{1}$ in an open set $\mathcal{U} \subset \mathbb{C}^{m+n}$. Then, for any $f \in \mathcal{C}^{1}(M)$, the following statements are equivalent:

(i) $f$ is a CR function on $M$;

(ii) $\bar{\partial}_{M}(f)=0$;

(iii) $\bar{\partial}\left(f[M]^{0, n}\right)=0$, i.e. $\int_{\mathcal{U} \cap M} f \bar{\partial} \varphi=0$ for all $\varphi \in \mathcal{D}^{m+n, m-1}(\mathcal{U})$;

(iv) $\bar{\partial}\left(\pi_{*}\left(\zeta\left[\Gamma_{f}\right]_{m+n, m}\right)\right)=\pi_{*}\left(\zeta \bar{\partial}\left[\Gamma_{f}\right]^{0, n}\right)=0$.

Pr o of. Equivalence of (i) and (ii) is obvious. To prove that (ii) implies (iii), it results from $\bar{\partial}_{M}(f)=0$ that $f \bar{\partial} \varphi=\bar{\partial}(f \varphi)=d(f \varphi)$, since $\partial(f \varphi)=0$ by bidegree considerations, hence by Stokes' formula, $\int_{M} f \bar{\partial} \varphi=\int_{M} d(f \varphi)$ $=0$. The converse is obtained by choosing suitable forms $\varphi$. To prove that (iii) is equivalent to (iv), notice that obviously $\bar{\partial}\left(f[M]^{0, n}\right)=\bar{\partial}\left(\pi_{*}\left(\zeta \bar{\partial}\left[\Gamma_{f}\right]^{0, n}\right)\right)$.

The proof of Proposition 2.5 is complete.

3. CR meromorphic mappings. The natural generalization of meromorphy to CR category must include the appearance of indeterminacy points, not only being smooth $\mathrm{CR}$ from $M$ generic to $\mathbb{P}^{1}(\mathbb{C})$ or to a projective algebraic manifold $Y$. The following definition was devised by Harvey and Lawson and appears to be adequately large, but sufficiently stringent to maintain the possibility of filling a scarred maximally complex cycle with a holomorphic chain.

3.1. Definition ([5], [19]). Let $M$ be a $\mathcal{C}^{r}$-scarred generic submanifold of $\mathbb{C}^{m+n}$. Then a $C R$ meromorphic mapping is a $\mathcal{C}^{r}$-scarred CR mapping $\left(f, \mathcal{D}_{f},\left[\Gamma_{f}\right]\right)$ with values in a projective manifold $Y$.

By definition, a CR meromorphic mapping takes values in a projective algebraic manifold. In particular, if $Y=\mathbb{P}^{1}(\mathbb{C})$, the closure $\Gamma_{f}$ of the graph of 
$f$ over $\mathcal{D}_{f}$ defines a $\mathcal{C}^{r}$-scarred $\mathrm{CR}$ manifold of type $(m, n)$ in $\mathbb{C}^{m+n} \times \mathbb{P}^{1}(\mathbb{C})$ satisfying $d\left[\Gamma_{f}\right]=0$. Since any projective $\mathbb{P}^{k}(\mathbb{C})$ is birationally equivalent to a product of $k$ copies of $\mathbb{P}^{1}(\mathbb{C})([5])$, we can set $Y=\mathbb{P}^{1}(\mathbb{C})$ without loss of generality.

REMARK. We mention that a map defined on a dense open set $\mathcal{U} \subset \mathbb{C}^{m+n}$ with values in $\mathbb{P}^{1}(\mathbb{C})$ is meromorphic over $\mathcal{U}$ if and only if the closure $\Gamma_{f}$ of its graph $\left\{(p, f(p)) \in \mathcal{U} \times \mathbb{P}^{1}(\mathbb{C})\right\}$ defines a $\mathcal{C}^{r}$-scarred complex submanifold of $\mathcal{U} \times \mathbb{P}^{1}(\mathbb{C})$. This justifies in a certain sense the above definition.

Let $\left(t_{1}, \ldots, t_{m+n},\left[\zeta_{0}: \zeta_{1}\right]\right)=(t, \zeta)$ denote coordinates on $\mathbb{C}^{m+n} \times \mathbb{P}^{1}(\mathbb{C})$ and let $\pi: \mathbb{C}^{m+n} \times \mathbb{P}^{1}(\mathbb{C}) \rightarrow \mathbb{C}^{m+n}$ denote the projection onto the first factor.

3.2. Definition ([19]). A point $p \in M$ is called an indeterminacy point if $\{p\} \times \mathbb{P}^{1}(\mathbb{C}) \subset \Gamma_{f}$. Denote by $\Sigma_{f}=\left\{p \in M:\{p\} \times \mathbb{P}^{1}(\mathbb{C}) \subset \Gamma_{f}\right\}$ the indeterminacy locus of $f$.

The following two propositions are due to Sarkis [19]. The first one is a clever remark about thinness of the indeterminacy set $\Sigma_{f}$. We present his proof for completeness.

3.3. Proposition (Sarkis [19]). Let $M$ be a $\mathcal{C}^{1}$-scarred $C R$ manifold of type $(m, n)$ in $\mathbb{C}^{m+n}$ and let $\left(f, \mathcal{D}_{f},\left[\Gamma_{f}\right]\right)$ be a $C R$ meromorphic mapping on M. Then:

(i) For almost all $a \in \mathbb{P}^{1}(\mathbb{C})$, the level set $\Lambda_{a}=\pi\left(\{\zeta=a\} \cap \Gamma_{f}\right)$ is a $\mathcal{C}^{1}$-scarred 2-codimensional submanifold of $M$;

(ii) For every such a, the indeterminacy set $\Sigma_{f}$ is a closed subset of $\Lambda_{a}$ with empty interior.

P r o of. We begin by asserting that for almost all complex $(m+n)$-dimensional linear subspaces $H$ of $\mathbb{C}^{m+n} \times \mathbb{P}^{1}(\mathbb{C})$, we have: 1) $\mathcal{H}^{d-2}\left(\Gamma_{f} \cap H\right)$ $<\infty$ and 2) $\Gamma_{f}^{H}:=\Gamma_{f} \cap H$ is a $\mathcal{C}^{1}$-scarred $(2+n)$-codimensional real submanifold of $H$. This follows by known facts from geometric measure theory (see [5]). After a small linear change of coordinates in $\mathbb{C}^{m+n} \times \mathbb{P}^{1}(\mathbb{C})$, this holds for almost every $a \in \mathbb{P}^{1}(\mathbb{C})$ with $H=H_{a}=\{\zeta=a\}$. Write $\Gamma_{f}^{a}=H_{a} \cap \Gamma_{f}$. Obviously, $\Gamma_{f}^{a} \subset M \times\{a\}$ is a $\mathcal{C}^{1}$-scarred submanifold in $H_{a}$ if and only if $\Gamma_{f}^{a}$ is a $\mathcal{C}^{1}$-scarred 2-codimensional submanifold of $M$. This gives (i).

Assume by contradiction that $\Sigma_{f}$ contains a nonempty open set $\mathcal{L} \subset$ $\operatorname{Reg}\left(\Lambda_{a}\right)$, so $\mathcal{L} \times \mathbb{P}^{1}(\mathbb{C}) \subset \Gamma_{f}$. For dimensional reasons, $\mathcal{L} \times \mathbb{P}^{1}(\mathbb{C}) \equiv \Gamma_{f}$ there. Indeed, $\operatorname{dim}_{\mathbb{R}}\left(\mathcal{L} \times \mathbb{P}^{1}(\mathbb{C})\right)=2+\operatorname{dim}_{\mathbb{R}} \mathcal{L}=\operatorname{dim}_{\mathbb{R}} \Gamma_{f}$. Let $p_{0} \in \mathcal{L}$. That $\Gamma_{f}$ is vertical over $\mathcal{L}$ near $\operatorname{Reg}\left(\Lambda_{a}\right)$ is impossible, since $\left.\Gamma_{f}\right|_{\mathcal{D}_{f}}$ is a $\mathcal{C}^{1}$-smooth graph over the dense open set $\mathcal{D}_{f} \subset M$ whose closure contains $p_{0}$.

The proof of Proposition 3.3 is complete. 
REMARK. The small linear change of coordinates above was necessary, since all the $H_{a}$ can be contained in the thin set of $H$ where neither 1) nor 2) holds.

A classical observation is that with each pair consisting of a volume form $d \lambda_{M}$ on an oriented $\mathcal{C}^{r}$-scarred CR manifold $M$ and an integrable function on $M$ there is associated a distribution $T_{f}$ in a natural way by $\left\langle T_{f}, \varphi\right\rangle=\int_{U} f \varphi d \lambda_{M}$. However, $T_{f}$ depends on $d \lambda_{M}$. The transpose operator ${ }^{\tau} \bar{L}$ of a $\mathrm{CR}$ vector field $\bar{L} \in \Gamma\left(U, T^{0,1} M\right)$ with respect to $d \lambda_{M}$ is defined by $\int_{M} \varphi \bar{L}(\psi) d \lambda_{M}=\int_{M}{ }^{\tau} \bar{L}(\varphi) \psi d \lambda_{M}$ for all functions $\varphi$, $\psi$ with compact support. Then $T_{f}$ is CR if and only if $\left\langle T_{f},{ }^{\tau} \bar{L}(\varphi)\right\rangle=0$ if and only if $f$ is CR. A distribution $T$ on $M$ is called a $C R$ distribution if $\left\langle T,{ }^{\tau} \bar{L}(\varphi)\right\rangle=0$ for all $\varphi \in \mathcal{D}(M)$. Although ${ }^{\tau} \bar{L}$ depends on the choice of $d \lambda_{M}$, this annihilating condition is independent. Indeed, given $d \lambda_{M}^{1}$ and $d \lambda_{M}^{2}$, there always exists a function $a \in \mathcal{C}^{\infty}\left(M, \mathbb{C}^{*}\right)$ with $d \lambda_{M}^{2}=a d \lambda_{M}^{1}$, so $\tau^{2} \bar{L}(\varphi)=a^{-1} \tau^{1} \bar{L}(a \varphi)$, whence the equivalence by linearity of distributions.

The statement below and its proof are known if $\operatorname{Sc}(M)=\emptyset$, i.e. $f$ is $\mathcal{C}^{1}$; here, the condition $d\left[\Gamma_{f}\right]$ helps in an essential way to keep it true in the $\mathcal{C}^{1}$-scarred category.

3.4. Proposition (Sarkis [19]). Let $M$ be a $\mathcal{C}^{1}$-scarred $C R$ manifold of type $(m, n)$ in $\mathbb{C}^{m+n}$, let $\left(f, \mathcal{D}_{f},\left[\Gamma_{f}\right]\right)$ be a $C R$ meromorphic mapping on $M$ and let $\Sigma_{f}=\left\{p \in M:\{p\} \times \mathbb{P}^{1}(\mathbb{C}) \subset \Gamma_{f}\right\}$. Then there exists an order zero $C R$ distribution $T_{f}$ on $M \backslash \Sigma_{f}$ such that $\left.T_{f}\right|_{\mathcal{D}_{f}} \equiv f$. In a chart $(U, \mathbb{C})$ of $M \times \mathbb{P}^{1}(\mathbb{C})$ with $\left.(U \times\{\infty\}) \cap \Gamma_{f}\right|_{U}=\emptyset$, given a volume form $d \lambda_{M}$ on $M \backslash \operatorname{Sc}(M), T_{f}$ is defined by

$$
\left[\Gamma_{f}\right]\left(\zeta \pi_{*}\left(\varphi d \lambda_{M}\right)\right)=\int_{\Gamma_{f}} \zeta \pi^{*}\left(\varphi d \lambda_{M}\right) \quad \text { for all } \varphi \in \mathcal{C}_{\mathrm{c}}^{\infty}(U) .
$$

Proof. As before, $\pi: U \times \mathbb{C} \rightarrow U$ denotes $(z, \zeta) \mapsto z$. By assumption, $U \subset M \backslash \Sigma_{f}$. Since $U \times\left.\{\infty\} \cap \Gamma_{f}\right|_{U}=\emptyset$, one has $\sup _{\left.\zeta \in \Gamma_{f}\right|_{U}}|\zeta|<\infty$. Let $V \Subset U$ be open with $\bar{V}$ compact and let $\varphi \in \mathcal{C}_{\mathrm{c}}^{\infty}(V)$. Then

$$
\left.\left|\left\langle T_{f}, \varphi\right\rangle\right|=\mid \Gamma_{f}\right]\left(\zeta \pi^{*}\left(\varphi d \lambda_{M}\right)\right)\left|\leq \sup _{\zeta \in \Gamma_{f} \mid U}\right| \zeta \mid \mathcal{H}^{d}\left(\Gamma_{f} \cap\left(V \times \mathbb{P}^{1}(\mathbb{C})\right)\right)\|\varphi\|_{L^{\infty}(U)}
$$

$(d=\operatorname{dim} M)$, which proves that $T_{f}$ is a distribution of order zero over $U$ $\left(\left.T_{f}\right|_{U} \in L_{\mathrm{loc}}^{\infty}(M)\right)$.

$T_{f}$ is clearly equal to the distribution associated with $f$ on the open dense set $\mathcal{D}_{f} \subset M$ where $f$ is $\mathcal{C}^{1}$. Indeed,

$$
\forall \varphi \in \mathcal{C}_{\mathrm{c}}^{\infty}(U), \quad\left\langle T_{f}, \varphi\right\rangle=\int_{\Gamma_{f} \cap \pi^{-1}(U)} \zeta \pi^{*}\left(\varphi d \lambda_{M}\right)=\int_{U} f \varphi d \lambda_{M}=\langle f, \varphi\rangle .
$$


Let now $\bar{L} \in \Gamma\left(U, T^{0,1} M\right)$ and complete the pair $\left(L^{*}, \bar{L}^{*}\right)$ to a basis $\left(L^{*}, \bar{L}^{*}, L_{2}^{*}, \bar{L}_{2}^{*}, \ldots, L_{m}^{*}, \bar{L}_{m}^{*}, \lambda_{1}^{*}, \ldots, \lambda_{n}^{*}\right)$ of $T^{*} M$, so that, furthermore,

$$
d \lambda_{M}=(i / 2)^{m} L^{*} \wedge \bar{L}^{*} \wedge L_{2}^{*} \wedge \bar{L}_{2}^{*} \wedge \ldots \wedge L_{m}^{*} \wedge \bar{L}_{m}^{*} \wedge \lambda_{1}^{*} \wedge \ldots \wedge \lambda_{n}^{*} .
$$

By Stokes' formula, $\int_{U} \varphi \bar{L}(\psi) d \lambda_{M}=-\int_{U} \bar{L}(\varphi) \psi d \lambda_{M}$ for all $\varphi, \psi \in \mathcal{C}_{c}^{\infty}(U)$, so that the transpose ${ }^{\tau} \bar{L}$ equals $-\bar{L}$ in the above chosen frame. One must prove that $\left\langle T_{f}, \bar{L}(\varphi)\right\rangle=0$ for all $\varphi \in \mathcal{C}_{c}^{\infty}(U)$. To do so, notice that by introducing the $(2 m+n-1)$-form $d \mu_{M}=(i / 2)^{m} L^{*} \wedge L_{2}^{*} \wedge \bar{L}_{2}^{*} \wedge \ldots \wedge \lambda_{n}^{*}$, one has

$$
\bar{L}(\varphi) d \lambda_{M}=\bar{\partial}_{M}\left(\varphi d \mu_{M}\right)=\bar{\partial}\left(\varphi d \mu_{M}\right)
$$

on $M$, since $\bar{\partial}_{M}=\sum_{j=1}^{m} \bar{L}_{j}(\cdot) \bar{L}_{j}^{*}$ and $\left.\bar{\partial}\right|_{M}=\bar{\partial}_{M}$. Therefore,

$$
\left\langle T_{f}, \bar{L}(\varphi)\right\rangle=\left[\Gamma_{f}\right]\left(\zeta \pi^{*}\left(\bar{\partial}\left(\varphi d \mu_{M}\right)\right)\right)=\left[\Gamma_{f}\right]^{0, n}\left(\bar{\partial}\left(\zeta \pi^{*}\left(\varphi d \mu_{M}\right)\right)\right)=0,
$$

by the above-noticed fact that $\bar{\partial}\left[\Gamma_{f}\right]^{0, n}=0$ and since $\bar{\partial}\left(\zeta \pi^{*}\left(\varphi d \mu_{M}\right)\right)$ is an $(m+n, m)$-form on $U \times \mathbb{C}$.

The proof of Proposition 3.4 is complete.

REMARK. In fact, $f$ induces an intrinsic CR current $\left[C_{f}\right]$ on $M \backslash \Sigma_{f}$ by $\left[C_{f}\right](\alpha)=\left[\Gamma_{f}\right]\left(\zeta \pi^{*} \alpha\right)$ in a chart as above. CR distributions will be more concrete for the properties of extendability.

4. Local extension of CR meromorphic mappings. Let $\Omega$ be a bounded domain with connected $\mathcal{C}^{1}$ boundary in $\mathbb{C}^{n}$ where $n \geq 3$, and let $Y$ be a projective manifold. Harvey and Lawson proved that any $\mathcal{C}^{1}$ scarred mapping $f: b \Omega \rightarrow Y$ which satisfies the tangential Cauchy-Riemann equations at the regular points of $f$ and such that $d\left[\Gamma_{f}\right]=0$ in the sense of currents extends to a meromorphic map $F: \Omega \rightarrow Y$. By considering the graph of $f$ over $b \Omega$, it is a corollary of the following extension theorem.

Theorem (Harvey-Lawson [5]). Let $(V, b V)$ be a compact, complex, $p$ dimensional subvariety with boundary in $\mathbb{P}^{n}(\mathbb{C}) \backslash \mathbb{P}^{n-q}(\mathbb{C})$, where $b V$ is a scarred $\mathcal{C}^{1}$-cycle whose regular points form a connected open set. If $p>2 q$, then every scarred $C R$ map of class $\mathcal{C}^{1}$ carrying $b V$ into a projective manifold $Y$ extends to a meromorphic map $F: V \rightarrow Y$.

The case $\operatorname{dim}_{\mathbb{R}}(b \Omega)=3$ and $b \Omega \mathcal{C}^{2}$ follows from the work of DolbeaultHenkin [4].

TheOREM (Dolbeault-Henkin [4]). Let $\Omega$ be a bounded domain in $\mathbb{C}^{2}$ with $b \Omega$ of class $\mathcal{C}^{2}$. Then every $\mathcal{C}^{2}$-smooth $C R$ mapping $b \Omega \rightarrow \mathbb{P}^{1}(\mathbb{C})$ admits a meromorphic extension to $\Omega$.

In a forthcoming paper, Sarkis generalizes the above result allowing indeterminacies for $f \mathrm{CR}$ meromorphic and a holomorphically convex compact set $K$, in the spirit of Lupacciolu ([12]). 
TheOREM (Sarkis [19]). Let $\Omega$ be a relatively compact domain in a Stein manifold $\mathcal{M}, \operatorname{dim} \mathcal{M} \geq 2$, let $K=\widehat{K}_{\mathcal{H}(\mathcal{M})}$ be a holomorphically convex compact set and assume that $b \Omega \backslash K$ is a connected $\mathcal{C}^{1}$-scarred hypersurface in $\mathcal{M} \backslash K$. Then any $C R$ meromorphic mapping $\left(f, \mathcal{D}_{f},\left[\Gamma_{f}\right]\right)$ on $b \Omega \backslash K$ admits a unique meromorphic extension to $\Omega \backslash K$.

We mention that the above theorem is known for $f \mathrm{CR} \mathcal{C}^{1}$ or CR meromorphic $\mathcal{C}^{1}$ without indeterminacies, by other methods $([18])$; see Theorem 4.2 below.

Global and local extension theorems. A general feature of global extension of $\mathrm{CR}$ functions is that in many cases two independent steps must be made:

I. Prove that $\mathrm{CR}(M)$ extends holomorphically (meromorphically) to a one-sided neighborhood $\mathcal{V}^{b}(M)$ (here, $M$ is a $\mathcal{C}^{1}$-scarred hypersurface);

II. Prove that the envelope of holomorphy (meromorphy) of $\mathcal{V}^{b}(M)$ contains a large open set, e.g. $\Omega$ if $M=b \Omega$.

Step II is known to be equivalent in both cases: the envelope of holomorphy and the envelope of meromorphy of an open set coincide.

THEOREM (Ivashkovich [6]). Let $Y$ be a compact Kähler manifold and $f$ a meromorphic map from a domain $\Omega$ in some Stein manifold into $Y$. Then $f$ extends to a meromorphic map from the envelope of holomorphy $\widehat{\Omega}$ of $\Omega$ into $Y$.

Thus, every positive global extension theorem about CR functions extends to a result about meromorphic CR mappings, provided one can prove by local techniques that they extend meromorphically to open sets $\mathcal{V}^{b}(M)$ attached to real submanifolds $M \subset \mathbb{C}^{m+1}$. Indeed, the size of $\widehat{\mathcal{V}^{b}}(M)$ can be studied by means of global techniques, e.g. integral formulas. In most cases, including special results in partially convex-concave manifolds, the disc envelope of such $M$ will contain some attached open one-sided neighborhoods $\mathcal{V}^{b}(M)$ or wedges $\mathcal{W}$ with edge $M$.

In this direction, a classical result is the Hartogs-Levi theorem: Let $\Omega \Subset$ $\mathbb{C}^{n}, n \geq 2$, be a bounded domain and let $\mathcal{V}(b \Omega)$ be an open neighborhood of its boundary. Then holomorphic (meromorphic) functions on $\mathcal{V}(b \Omega)$ extend holomorphically (meromorphically) to $\Omega$.

Therefore, it is of great importance to answer the question of Henkin and Sarkis (which was not raised by Harvey and Lawson in 1977): Is there a local version of the meromorphic extension phenomenon? (e.g. a Lewy extension phenomenon). If the $\mathrm{CR}$ meromorphic mapping $\left(f, \mathcal{D}_{f},\left[\Gamma_{f}\right]\right)$ does not have indeterminacies, then it is locally $\mathrm{CR}$, so the answer is positive. We mention, however, that the most natural notion of CR meromorphic maps is the one where indeterminacies really occur (see Definitions 3.1 and 3.2). 
Thus, a satisfactory understanding of CR meromorphy involves local extension theory and various removable singularities theorems ([8], [11], [15], [17], [16]). This paper is devoted to delineating some.

CR meromorphy and removable singularities. Let $M$ be a piece of a generic submanifold of $\mathbb{C}^{m+n}$. The local holomorphic extension phenomenon for $\mathrm{CR}(M)$ and $\mathcal{D}_{\mathrm{CR}}^{\prime}(M)$ as well arises at most points of $M$, according to the theory of Trépreau and Tumanov.

By a wedge of edge $M$ at $p_{0} \in M$, we mean an open set in $\mathbb{C}^{m+n}$ of the form

$$
\mathcal{W}=\{z+\eta: z \in U, \eta \in C\}
$$

for some open neighborhood $U$ of $p_{0}$ in $M$ and some convex truncated open cone $C$ in $T_{p_{0}} \mathbb{C}^{m+n}$, i.e. the intersection of a convex open cone with a ball centered at 0 .

$M$ is called minimal at $p_{0}$ if the following property is satisfied.

Theorem (Trépreau, $n=1$; Tumanov, $n \geq 2$ ). Assume $M \subset \mathbb{C}^{m+n}$ is generic, $\mathcal{C}^{2, \alpha}(0<\alpha<1), \mathrm{CRdim} M=m \geq 1, \operatorname{codim} M=n \geq 1$ and let $p \in M$. Then there exists a wedge $\mathcal{W}_{p}$ of edge $M$ at $p$ such that $\mathrm{CR}(M), L_{\mathrm{loc}, \mathrm{CR}}^{1}(M), L_{\mathrm{loc}, \mathrm{CR}}^{\infty}(M), \mathcal{D}_{\mathrm{CR}}^{\prime}(M)$ extend holomorphically to $\mathcal{W}_{p}$ if and only if there does not exist a CR manifold $S \subset M$ with $S \ni p$ and CRdim $S=$ CRdim $M$.

By Proposition 3.4, all components of CR meromorphic mappings $\left(f, \mathcal{D}_{f},\left[\Gamma_{f}\right]\right)$ on $M$ behave locally like a $\mathrm{CR}$ distribution outside the thin set $\Sigma_{f}$ of their indeterminacies, therefore extendability properties hold everywhere outside $\Sigma_{f}$ if $M$ is minimal at every point. Thus, to extend $f$ along steps I and II, one is naturally led to the problem of propagating holomorphic extension up to wedges over $\Sigma_{f}$. It appears that $\Sigma_{f}$ has size small enough to be coverable by wedges. Namely, in the hypersurface case, which has been intensively studied, all the necessary results are already known: A wedge attached to $M \backslash \Phi, \Phi \subset M$ closed, $\operatorname{codim} M=1$, is simply an open set $\mathcal{V}^{b}(b= \pm)$ containing at each point of $M \backslash \Phi$ a one-sided neighborhood of $M$ such that Int $\overline{\mathcal{V}^{b}}=\mathcal{V}^{b}$. An open connected set $\mathcal{W}_{0}$ is called a wedge attached to $M \backslash \Phi$ if there exists a continuous section $\eta: M \rightarrow T_{M} \mathbb{C}^{m+n}$ of the normal bundle to $M$ and $\mathcal{W}_{0}$ contains a wedge $\mathcal{W}_{p}$ of edge $M$ at $(p, \eta(p))$ for every $p \in M$. A closed set $\Phi \subset M$ is called $\mathcal{W}$-removable $\left(\mathcal{V}^{b}\right.$-removable if $n=1)$ if, given a wedge $\mathcal{W}_{0}$ attached to $M \backslash \Phi$, there exists a wedge $\mathcal{W}$ attached to $M$ with holomorphic functions in $\mathcal{W}_{0}$ extending holomorphically to $\mathcal{W}$.

Jöricke in the $\mathcal{C}^{2}$-smooth case and then Chirka-Stout weakening the smoothness assumption, using the profound solution by Shcherbina of the three-dimensional Cauchy-Riemann Dirichlet problem with continuous data, showed: 
Theorem (Jöricke, $\mathcal{C}^{2},[8]$; Chirka-Stout [2]). Let $M$ be a locally Lipschitz graphed hypersurface in $\mathbb{C}^{m+1}$, and let $\Sigma \subset M$ be a closed subset with empty interior of a $\mathcal{C}^{1}$-scarred two-codimensional submanifold $\Lambda \subset M$. Then $\Sigma$ is $\mathcal{V}^{b}$-removable.

In the greater codimensional case also, to prove local extension of CR meromorphic mappings one has in a natural way to prove $\mathcal{W}$-removability of $\Sigma_{f}$. Denote by $\operatorname{Sc}\left(\Sigma_{f}\right)$ the scar set of a scarred manifold $\Lambda$ which contains the indeterminacy set by Section 3 .

The main result of this paper is the following.

4.1. TheOREM. Let $M$ be a smooth generic manifold in $\mathbb{C}^{m+n}$, CRdim $M$ $=m \geq 1, \operatorname{codim} M=n \geq 1$, and assume that $M$ is minimal at every point of $M$. Then there exists a wedge $\mathcal{W}_{0}$ attached to $M$ such that all $C R$ meromorphic mappings $\left(f, \mathcal{D}_{f},\left[\Gamma_{f}\right]\right)$ extend meromorphically to $\mathcal{W}_{0}\left(\Sigma_{f}\right.$ is $\mathcal{W}$-removable) under the following circumstances:

(i) $n=1$ (hypersurface case), $M$ is $\mathcal{C}^{2, \alpha}$ and (only) globally minimal:

(ii) $M$ is $\mathcal{C}^{2, \alpha}$ and $\mathcal{H}^{d-3}\left(\operatorname{Sc}\left(\Sigma_{f}\right)\right)=0$;

(iii) $M$ is $\mathcal{C}^{\omega}$ (real-analytic).

Remark. The wedge $\mathcal{W}_{0}$ is universal: it does not depend on $\left(f, \mathcal{D}_{f},\left[\Gamma_{f}\right]\right)$.

REMARK. The smoothness assumptions make Theorem 4.1 weaker in the hypersurface case than the local meromorphic extension theorem that follows from the theorem of Jöricke-Chirka-Stout or than the global theorem of Sarkis. Nonetheless, $M$ need not be minimal at every point: see Lemma 4.4 below.

Applications: global meromorphic extension. In the following results, it is known that $\mathcal{V}^{b}(M)$ for $M=b \Omega, M=b \Omega \backslash \widehat{K}_{\bar{\Omega}}$ contain $\Omega, \Omega \backslash \widehat{K}_{\bar{\Omega}}$ respectively ([11], [17]). In the meromorphic case they were proved by Sarkis ([19], see also [12], [13], [9], [17]).

4.2. Theorem. Let $\Omega \Subset \mathbb{C}^{m+1}$ be a $\mathcal{C}^{2}$-bounded domain. Then any $C R$ meromorphic mapping $\left(f, \mathcal{D}_{f},\left[\Gamma_{f}\right]\right)$ on $b \Omega$ with values in $\mathbb{P}^{1}(\mathbb{C})$ extends meromorphically to $\Omega$.

4.3. Theorem. Let $\Omega \Subset \mathbb{C}^{2}$ be a $\mathcal{C}^{2}$-bounded domain and let $K \subset b \Omega$ be a compact set. Then any $C R$ meromorphic mapping on $b \Omega \backslash K$ with values in $\mathbb{P}^{1}(\mathbb{C})$ extends meromorphically to $\Omega \backslash \widehat{K}_{\bar{\Omega}}$, where $\widehat{K}_{\bar{\Omega}}=\{p \in \bar{\Omega}:|f(z)| \leq$ $\max _{K}|f|$ for all $\left.f \in \mathcal{H}(\mathcal{V}(\bar{\Omega}))\right\}$.

REMARK. In the above two theorems, the hypersurface $M=b \Omega$ need not be everywhere minimal: $\mathrm{CR}(M)$ automatically extend holomorphically to some $\mathcal{V}^{b}(M)$, since $M$ is known to be a single $C R$ orbit ([9]). To explain the phenomenon, we need some definitions. 
Let $M$ be a $\mathcal{C}^{2}$-smooth $\mathrm{CR}$ manifold. The $C R$ orbit of a point $p \in M$ is the set of all endpoints of piecewise smooth integral curves of $T^{c} M$ with origin $p$. The CR orbits partition $M$. Sussmann (see [21], [11]) showed that each $\mathrm{CR}$ orbit $\mathcal{O}_{\mathrm{CR}}$ has the structure of a smooth $\mathcal{C}^{1}$ manifold making the inclusion $\mathcal{O}_{\mathrm{CR}} \rightarrow M$ an injective $\mathcal{C}^{1}$ immersion. By construction, each $\mathcal{O}_{\mathrm{CR}}$ is a $\mathrm{CR}$ manifold with $\mathrm{CR} \operatorname{dim} \mathcal{O}_{\mathrm{CR}}=\mathrm{CR} \operatorname{dim} M$. Each $\mathrm{CR}$ manifold as $\mathcal{O}_{\mathrm{CR}}$ is locally embeddable as a generic submanifold of some $\mathbb{C}^{N}, N \leq m+n$. A CR manifold $M$ is called globally minimal if $M$ consists of a single CR orbit.

The relevance of $\mathrm{CR}$ orbits to the extendability properties of CR functions is due to Trépreau and yields the following finest possible extension theorem:

Theorem ([21], [22], [9], [14], [17]). If $M$ is a globally minimal locally embeddable generic $\mathcal{C}^{2, \alpha}$-smooth $(0<\alpha<1)$ manifold, then there exists a wedge $\mathcal{W}_{0}$ attached to $M$ such that $\mathrm{CR}(M), L_{\mathrm{loc}, \mathrm{CR}}^{1}(M), L_{\mathrm{loc}, \mathrm{CR}}^{\infty}(M)$, $\mathcal{D}_{\mathrm{CR}}^{\prime}(M)$ all extend holomorphically to $\mathcal{W}_{0}$.

Proof of Theorems 4.2 and 4.3. In the hypersurface case, thin sets such as $\Sigma_{f}$ do not perturb CR orbits:

4.4. Lemma. Let $M$ be a $\mathcal{C}^{2}$ hypersurface in $\mathbb{C}^{m+1}$ and let $\Sigma$ be a closed subset with nonempty interior of some $\mathcal{C}^{1}$-scarred two-codimensional submanifold $\Lambda \subset M$. Then, for all CR orbits $\mathcal{O}_{\mathrm{CR}} \subset M, \mathcal{O}_{\mathrm{CR}} \backslash\left(\mathcal{O}_{\mathrm{CR}} \cap \Sigma\right)$ is a single $C R$ orbit of $M \backslash \Sigma$.

Pro of. The real dimension of an $\mathcal{O}_{\mathrm{CR}}$ is $\geq 2 m$ and $\leq 2 m+n=2 m+1$ if $n=1$. So $\Sigma$ is too small to make obstruction to an orbit. However, the lemma can fail in codimension $\geq 2$.

Thus, Theorems 4.2 and 4.3 rely on the following properties.

Proposition. Let $M=b \Omega$ or $b \Omega \backslash \widehat{K}_{\bar{\Omega}}$, codim $M=1, M \mathcal{C}^{2}$ and let $\left(f, \mathcal{D}_{f},\left[\Gamma_{f}\right]\right)$ be a $C R$ meromorphic mapping on $M$. Then

(i) $M$ is a single $C R$ orbit ([10]);

(ii) $M \backslash \Sigma_{f}$ is a single $C R$ orbit, hence $f$ extends meromorphically to $\mathcal{V}^{b}\left(M \backslash \Sigma_{f}\right)$

(iii) $\Sigma_{f}$ is $\mathcal{V}^{b}$-removable, hence $f$ extends meromorphically to $\mathcal{V}^{b}(M)$.

One then deduces 4.2 and 4.3 with the Ivashkovich theorem.

$\mathcal{W}$-removability. Theorem 4.1 is reduced to the $\mathcal{W}$-removability of $\Sigma_{f}$. By Proposition 3.3, $\Sigma_{f}$ is a closed subset with empty interior of some $\mathcal{C}^{1}$ scarred two-codimensional submanifold $\Lambda \subset M, \Sigma_{f} \subset \operatorname{Sc}(\Lambda) \cup \operatorname{Reg}(\Lambda)$. Write $\Sigma_{f}=E \cup \Phi, \Phi=\operatorname{Reg}(\Lambda) \cap \Sigma_{f}, E=\operatorname{Sc}(\Lambda) \cap \Sigma_{f}, \mathcal{H}^{d-2}(E)=0 . \Phi$ is already known to be removable. 
Theorem ([15], [16]). Let $M$ be a $\mathcal{C}^{2, \alpha}$-smooth $(0<\alpha<1)$ generic manifold in $\mathbb{C}^{m+n}$, minimal at every point, CRdim $M=m \geq 1$, and let $N \subset M$ be a connected $\mathcal{C}^{1}$-smooth submanifold with $\operatorname{codim}_{M} N=2$. Then every proper closed subset $\Phi \subset N$ is $\mathcal{W}$-removable.

The purpose of Section 5 is to establish:

4.5. TheOREM. Let $M$ be a $\mathcal{C}^{2, \alpha}$-smooth generic manifold in $\mathbb{C}^{m+n}$, globally minimal with $\mathrm{CR} \operatorname{dim} M=m \geq 1$. Then

(i) if $n=1$ or $M$ is $\mathcal{C}^{\omega}$, then any closed $E \subset M$ with $\mathcal{H}_{\text {loc }}^{2 m+n-2}(E)=0$ is $\mathcal{W}$-removable;

(ii) if $M$ is minimal at every point, then any closed subset $E \subset M$ with $\mathcal{H}_{\text {loc }}^{2 m+n-3}(E)=0$ is $\mathcal{W}$-removable.

Remark. Dinh and Sarkis obtained Theorem 4.5 assuming that $M$ is of type one in the sense of Bloom-Graham, i.e. the first order Lie brackets of vector fields in $T^{c} M$ generate $T M,\left[T^{c} M, T^{c} M\right]=T M$, for $M \mathcal{C}^{4}$ smooth $([3])$.

$L^{p}$-removability. Let $M$ be a locally embeddable CR manifold of class $\mathcal{C}^{2}$. A closed subset $\Phi$ of $M$ is called $L^{p}$-removable, $p \geq 1$, if each function $f \in L_{\text {loc }}^{p}(M)$ which satisfies the Cauchy-Riemann equations $L f=0$ (in the distribution sense) on $M \backslash \Phi$ satisfies the equation $L f=0$ on the whole of $M$, or, for short, if

$$
L_{\mathrm{loc}, \mathrm{CR}}^{p}(M \backslash \Phi) \cap L_{\mathrm{loc}}^{p}(M)=L_{\mathrm{loc}, \mathrm{CR}}^{p}(M) .
$$

The authors have proved in [16] that $L^{p}$-removability holds if $\mathcal{W}$-removability holds, for closed subsets $\Phi \subset M$ with $\mathcal{H}_{\text {loc }}^{d-2}(\Phi)<\infty$. Therefore:

4.6. TheOREm. Theorems 4.5 and 5.1 are true for $L^{p}$-removability, $1 \leq$ $p \leq \infty$

5. Removable singularities. As pointed out in Section 4, the relationships between extendability properties of CR functions and the geometry of a CR manifold are adequately reflected by its CR orbits. It is thus natural to state a removable singularities theorem in the most general context.

5.1. THEOREM. Let $M$ be a smooth generic globally minimal manifold in $\mathbb{C}^{m+n}$ with $\operatorname{CRdim} M=m \geq 1, \operatorname{codim} M=n \geq 1$ and $\operatorname{dim} M=d=2 m+n$. Then every closed subset $E$ of $M$ such that $M \backslash E$ is globally minimal is $\mathcal{W}$-removable under each of the following conditions:

(i) $n=1, M$ is $\mathcal{C}^{2, \alpha}$ and $\mathcal{H}^{d-2}(E)=0$;

(ii) $M$ is $\mathcal{C}^{2, \alpha}$ and $\mathcal{H}^{d-3}(E)=0$;

(iii) $M$ is $\mathcal{C}^{\omega}$. 
P r o of. Following the scheme of proof devised in [15] and [16], we present the development of the proof of (i), (ii) and (iii) in five essential steps. Let $E \subset M$ be closed with $\mathcal{H}^{d-2}(E)=0$.

SteP 1: Reduction to the removal of a point. By assumption, $M \backslash E$ is globally minimal. Then, according to the extension theorem, CR functions are wedge extendable at every point of $M \backslash E$. However, the direction of the wedges may have discontinuities. Fortunately, the edge of the wedge theorem enables one to fill in larger wedges by means of attached analytic discs at points of discontinuity. Therefore, there exists a wedge $\mathcal{W}_{0}$ attached to $M \backslash E$ to which $\mathrm{CR}(M)$ holomorphically extends.

Using a $\mathcal{C}^{2, \alpha}$-smooth partition of unity on $M \backslash E$, we can deform $M$ inside $\mathcal{W}_{0}$ over $M \backslash E$ into a $\mathcal{C}^{2, \alpha}$-smooth manifold $M^{d}$ ( $d$ here not to be confused with $\operatorname{dim} M)$. Then, instead of a function $f \in \operatorname{CR}(M \backslash E)$, we get a function $f$, holomorphic in a neighborhood $\omega\left(\equiv \mathcal{W}_{0}\right)$ of $M^{d} \backslash E$ in $\mathbb{C}^{m+n}$. The aim is now to prove that such holomorphic functions extend to a wedge $\mathcal{W}_{1}^{d}$ attached to $M^{d}$. The construction will depend smoothly on $d$, so that letting $d$ tend to zero, one obtains a wedge $\mathcal{W}_{1}$ attached to $M$ (for details, see Section 5 of [15]).

The first key point is that the continuity principle along analytic discs with boundaries in $\omega$ can now be exploited to show that the envelope of holomorphy of $\omega$ contains a wedge $\mathcal{W}_{1}^{d}$ attached to $M^{d}$.

Let $\Delta$ denote the unit disc in $\mathbb{C}$ and $b \Delta$ its boundary, the unit circle. An embedded analytic disc $A$ attached to $M$ is said to be analytically isotopic to a point in $M$ if there exists a $\mathcal{C}^{1}$-smooth mapping $(s, \zeta) \mapsto A_{s}(\zeta), 0 \leq s \leq 1$, $\zeta \in \bar{\Delta}$, such that $A_{0}=A$, each $A_{s}$ is an embedded analytic disc attached to $M$ for $0 \leq s<1$ and $A_{1}$ is a constant mapping $\bar{\Delta} \rightarrow\{\mathrm{pt}\} \in M$. Using Cauchy estimates and controlling connectedness, it is possible to prove (the embedding condition yields monodromy, [15], Proposition 3.2):

5.2. Proposition. Let $M$ be generic, $\mathcal{C}^{2, \alpha}$, let $\Phi$ be a proper closed subset of $M$ and let $\omega$ be a neighborhood of $M \backslash \Phi$ in $\mathbb{C}^{m+n}$. If an embedded disc $A$ attached to $M \backslash \Phi$ is analytically isotopic to a point in $M \backslash \Phi$, then there exists a neighborhood $\mathcal{V}(A(\bar{\Delta}))$ in $\mathbb{C}^{m+n}$ such that, for each function $f \in \mathcal{H}(\omega)$, there exists a function $F \in \mathcal{H}(\mathcal{V}(A(\bar{\Delta})))$ such that $F=f$ in a neighborhood of $A(b \Delta)$.

Call a point $p \in E \mathcal{W}$-removable if there exists a wedge $\mathcal{W}_{p}$ of edge $M^{d}$ at $p$ with $\mathcal{H}(\omega)$ extending holomorphically to $\mathcal{W}_{p}$.

Define

$$
\mathcal{A}=\{\Psi \subset E \text { closed : } M \backslash \Psi \text { is globally minimal and }
$$

$$
M \backslash \Psi \text { is } \mathcal{W} \text {-removable in } M \backslash \Psi\}
$$

and define $E_{\mathrm{nr}}=\bigcap_{\Psi \in \mathcal{A}} \Psi$, the nonremovable part of $E$. Then $M \backslash E_{\mathrm{nr}}$ is also 
globally minimal. By deforming $M^{d}$ into a manifold $\left(M^{d}\right)^{d_{1}}$ over $E \backslash E_{\mathrm{nr}}$, we can assume that we must remove $E_{\mathrm{nr}}$ for $\mathcal{H}\left(\mathcal{V}\left(\left(M^{d}\right)^{d_{1}} \backslash E_{\mathrm{nr}}\right)\right)$ instead of $E$. But $E_{\mathrm{nr}}$ is the smallest nonremovable subset of $E$ keeping $\left(M^{d}\right)^{d_{1}} \backslash E_{\mathrm{nr}}$ globally minimal. Assuming that $E_{\mathrm{nr}} \neq \emptyset$, we now reach a contradiction by showing that a point $p_{1} \in E_{\mathrm{nr}}$ is $\mathcal{W}$-removable. We write $E$ and $M$ instead of $\left(M^{d}\right)^{d_{1}}$. Thus, to prove Theorem 5.1, it is sufficient to prove that the new $E$ is removable near one of its points.

According to Lemma 2.3 of [16], the fact that $M \backslash E$ is globally minimal and the existence of chains of infinitesimally small analytic discs approximating integral curves of $T^{c} M$ insure the existence of a generic manifold $M_{1}$ of codimension one in $M$ through a point $p_{1} \in E$ such that $T_{p_{1}} M_{1} \not \supset T_{p_{1}}^{c} M$ and $E \subset M_{1}^{-}$, the closed negative side of $M_{1}$ in $M$, near $p_{1}$. Let us quote this (elementary) differential geometric statement as: Let $M$ be a $\mathcal{C}^{2}$ manifold, let $K \subset T M$ be a $\mathcal{C}^{1}$ subbundle, let $E \subset M$ be a closed nonempty set and assume that $M$ and $M \backslash E$ are both single $K$-orbits. Then there exists a point $p_{1} \in E$ and $a \mathcal{C}^{1}$ hypersurface $M_{1} \subset M$ with $p_{1} \in M_{1}, T_{p_{1}} M_{1} \not \supset K\left(p_{1}\right)$ and $E \subset M_{1}^{-}$near $p_{1}$.

Finally, by the definition of $\mathcal{A}$, and by disposition of $M_{1}, E \subset M_{1}^{-}$, it suffices to show that $p_{1}$ is $\mathcal{W}$-removable. Indeed, for a small neighborhood $\mathcal{V}\left(p_{1}\right)$ of $p_{1}$ in $M,(M \backslash E) \cup \mathcal{V}\left(p_{1}\right)$ is globally minimal, as $T_{p_{1}} M_{1} \not \supset T_{p_{1}}^{c} M$. Thus, to prove Theorem 5.1, it is sufficient to prove that a neighborhood of $p_{1} \in E$ is $\mathcal{W}$-removable.

STEP 2: Existence of a disc. Let $p_{1} \in E$ be as above and choose holomorphic coordinates $(w, z)=\left(w_{1}, \ldots, w_{m}, z_{1}, \ldots, z_{n}\right), z=x+i y$ on $\mathbb{C}^{m+n}$ such that $p_{1}=0, T_{0} M=\{x=0\}, T_{0}^{c} M=\{z=0\}, M$ is given by $n$ scalar equations $x=h(y, w)$, in vectorial notation, $h(0)=0, d h(0)=0$ and $M_{1}$ is given in $M$ by the supplementary equation $u_{1}=k\left(v_{1}, w_{2}, \ldots, w_{m}, y\right)$, for a $\mathcal{C}^{2}$-smooth $k$ with $k(0)=d k(0)=0$. We denote $M_{1}^{-}=\left\{u_{1} \leq\right.$ $\left.k\left(v_{1}, w_{2}, \ldots, w_{m}, y\right)\right\}$.

Our first construction of analytic discs attached to $M$ proceeds as follows.

5.3. Lemma ([16], Lemma 2.4). There exists an embedded analytic disc $A \in \mathcal{C}^{2, \beta}(\bar{\Delta})$ with $A(1)=p_{1}, A(b \Delta) \backslash\{1\} \subset M \backslash M_{1}^{-}$and $\left.\frac{d}{d \theta}\right|_{\theta=0} A\left(e^{i \theta}\right)=$ $v_{0} \in T_{p_{1}} M_{1}$.

It suffices to take, for small $\varrho_{1}>0$, the disc $A(\zeta)$ with $W_{\varrho_{1}}(\zeta)=$ $\left(\varrho_{1}(1-\zeta), 0, \ldots, 0\right)$ and with $Y$-component satisfying Bishop's equation $Y_{\varrho_{1}}=T_{1} h\left(Y_{\varrho_{1}}, W_{\varrho_{1}}\right)$ on $b \Delta$ (here, $T_{1}$ denotes the Hilbert transform $L^{2}(b \Delta)$ $\rightarrow L^{2}(b \Delta)$ vanishing at $\left.1,\left(T_{1} u\right)(1)=0\right)$.

Therefore, removability of $p_{1}$ will be a consequence of Proposition 5.4, proved below, and of Theorem 5.10 below. This is the main technical part of the article. This proposition provides extension outside a thin set $\mathcal{E}_{\Phi_{F}}$ which is studied below and which lives in an open (wedge) set $\mathcal{W}$ of $\mathbb{C}^{m+n}$. 
5.4. Proposition. Let $M$ be generic, $\mathcal{C}^{2, \alpha}$-smooth, let $p_{1} \in M$, let $E \subset$ $M$ be a closed subset with $\mathcal{H}_{\mathrm{loc}}^{d-2}(E)=0$, let $p_{1} \in E$, assume that there exists a one-codimensional generic $\mathcal{C}^{2}$-smooth manifold $M_{1} \subset M$ such that $E \subset M_{1}^{-}$and let $\omega$ be a neighborhood of $M \backslash E$ in $\mathbb{C}^{m+n}$. Let $A \in \mathcal{C}^{2, \beta}(\bar{\Delta})$ be a sufficiently small embedded analytic disc attached to $M, A(1)=p_{1}$, $\left.\frac{d}{d \theta}\right|_{\theta=0} A\left(e^{i \theta}\right)=v_{0} \in T_{p_{1}} M_{1}$, with $A(b \Delta \backslash\{1\}) \subset M \backslash M_{1}^{-}$. Then for each $\varepsilon>0$, there exist $v_{00} \in T_{p_{1}} M_{1}$ with $\left|v_{00}-v_{0}\right|<\varepsilon, v_{00} \notin T_{p_{1}}^{c} M$, a wedge $\mathcal{W}$ of edge $M$ at $\left(p_{1}, J v_{00}\right)$ and a closed set $\mathcal{E}_{\Phi_{E}}$ which is $\mathcal{C}^{2, \alpha}$ foliated by complex curves with $\mathcal{H}^{2 m+2 n-1}\left(\mathcal{E}_{\Phi_{E}}\right)=0$ such that for every holomorphic function $f \in \mathcal{H}(\omega)$ there exists a function $F \in \mathcal{H}\left(\omega \cup\left(\mathcal{W} \backslash \mathcal{E}_{\Phi_{E}}\right)\right)$ with $F=f$ in the intersection of $\mathcal{W} \backslash \mathcal{E}_{\Phi_{E}}$ with a neighborhood of $M \backslash E$ in $\mathbb{C}^{m+n}$.

REMARK. For any $e \geq 2$, we obtain statement 5.4 above for $\mathcal{H}^{d-e}(E)=0$ with $\mathcal{H}^{2 m+2 n-e+1}\left(\mathcal{E}_{\Phi_{E}}\right)=0\left(\mathcal{H}^{d-2}(E)=0\right.$ is crucial for isotopies, see 5.8 below).

SteP 3: Maximal families of analytic discs. This step consists in including the above analytic disc in a very large parameterized family of analytic discs obtained by varying the $W$-component, and its approximate radius, the base point $A(1)=p$ in a small neighborhood of 0 , and the point $A(-1)$ in $\omega([15],[16])$.

Let $\mu=\mu(y, w)$ be a $\mathcal{C}^{\infty}, \mathbb{R}$-valued function with support near the point $(y(-1), w(-1))$ that equals 1 there and let $\kappa: \mathbb{R}^{n} \rightarrow \mathbb{R}^{n}$ be a $\mathcal{C}^{\infty}$ function with $\kappa(0)=0$ and $\kappa^{\prime}(0)=\mathrm{Id}$. We can assume that the supports of $\mu$ and $\kappa$ are sufficiently concentrated in order that every manifold $M_{t}$ with equation

$$
x=H(y, w, t)=h(y, w)+\kappa(t) \mu(y, w)
$$

is contained in $\omega$ and the deformation is localized in a small neighborhood of $A(-1)$ in $\mathbb{C}^{m+n}$. Let $\chi=\chi(\zeta)$ be a smooth function on the unit circle supported in a small neighborhood of $\zeta=-1$.

We consider the disc with $W$-component

$$
\begin{aligned}
W_{\tau, a, \varrho, p}(\zeta)=\left(e^{i \tau}\left(\varrho_{1}-\varrho \zeta\right)+w_{1}^{0},(1-\zeta) a_{1} \varrho / \varrho_{1}+w_{2}^{0}, \ldots,\right. & \\
& \left.(1-\zeta) a_{m-1} \varrho / \varrho_{1}+w_{m}^{0}\right),
\end{aligned}
$$

where $a \in \mathbb{C}^{m-1}$ runs through a small neighborhood $\mathcal{A}$ of $0,0 \leq \varrho \leq \varrho_{1}$, $w^{0} \in \mathbb{C}^{m}, p \in M$ runs over a neighborhood of 0 and is represented by its coordinates $\left(w^{0}, y^{0}\right)$, and with $Y$-component $Y_{t, \tau, a, \varrho, p}$ which is the solution of Bishop's equation with parameters

$$
\begin{aligned}
Y_{t, \tau, a, \varrho, p}=T_{1} H\left(Y_{t, \tau, a, \varrho, p}, e^{i \tau}\left(\varrho_{1}-\varrho \zeta\right)+\right. & w_{1}^{0},(1-\zeta) a_{1} \varrho / \varrho_{1}+w_{2}^{0}, \ldots, \\
& \left.(1-\zeta) a_{m-1} \varrho / \varrho_{1}+w_{m}^{0}, t \chi\right)+y^{0},
\end{aligned}
$$

which exists and depends in a $\mathcal{C}^{2, \beta}$-smooth fashion on $(t, \tau, a, \varrho, p, \zeta)$, for all $0<\beta<\alpha$. Then $A_{t, \tau, a, \varrho, p}(1)=p$. When $\tau=0, a=0, \varrho=\varrho_{1}$ and $p=0$, we simply denote $A_{t, 0,0, \varrho_{1}, 0}$ by $A_{t}$. 
Let us recall that the normal deformations of $A$ near $A(-1)$ in $\omega$ can be chosen in such a way that the inner tangential direction $-\partial A_{t} / \partial \zeta(1)$ will describe a whole open cone in the normal bundle to $M$ at $A(1)=0$.

Let $\Pi$ denote the canonical bundle epimorphism $\Pi:\left.T \mathbb{C}^{m+n}\right|_{M} \rightarrow$ $\left.T \mathbb{C}^{m+n}\right|_{M} / T M$ and consider the $\mathcal{C}^{1, \beta}$ mapping

$$
D: \mathbb{R}^{n} \ni t \mapsto \Pi\left(-\frac{\partial A_{t}}{\partial \zeta}(1)\right) \in T_{0} \mathbb{C}^{m+n} / T_{0} M \simeq \mathbb{R}^{n} .
$$

We refer to [16] for a proof of the following.

5.5. Lemma (Tumanov [22]). $\chi$ can be chosen such that $\operatorname{rk} D^{\prime}(0)=n$.

This statement is more or less equivalent to the fact that the union of the discs describes a wedge of edge $M$ at 0 . We also have:

5.6. Lemma $([16]) \cdot \chi$ can be chosen such that the following holds: there exist $\tau_{0}>0$, a neighborhood $\mathcal{T}$ of 0 in $\mathbb{R}^{n}$ and a neighborhood $\mathcal{A}$ of 0 in $\mathbb{C}^{m-1}$ such that the set

$$
\Gamma_{0}=\left\{s \frac{d A_{t, \tau, \varrho_{1}, a, 0}}{d \theta}(1): s>0, t \in \mathcal{T}, \tau \in I_{\tau_{0}}, a \in \mathcal{A}\right\}
$$

is a $(2 m+n)$-dimensional open connected cone with vertex 0 in $T_{0} M$.

For convenience, we shall allow ourselves to shrink any open neighborhoods arising in the next constructions without explicit mention. By reasons of rank, the geometric meaning will be clear for sufficiently small parameters.

SteP 4: Isotopies. The main hypothesis so far is $\mathcal{H}^{d-2}(E)=0$. The boundaries of the analytic discs $A_{t, \tau, a, \varrho, p}(b \Delta)$ are embedded $\mathcal{C}^{2, \beta}$-smooth copies of $S^{1}$ in $M$, so one expects naturally that $A_{t, \tau, a, \varrho, p}(b \Delta) \cap E=$ $\emptyset$ generically. Furthermore, an isotopy property is required as stated in Proposition 5.2, in order to ensure that $\mathcal{H}(\omega)$ extends holomorphically to $\mathcal{V}\left(A_{t, \tau, a, \varrho, p}(\bar{\Delta})\right)$.

To prove an isotopy lemma, we recall briefly some facts concerning Hausdorff measures, taken from the very clear exposition of Chirka [1].

Let $E$ be an arbitrary subset of a metric topological space. For $\delta>0$, define

$\mathcal{H}_{\delta}^{s}(E)=\inf \left\{\sum_{j=1}^{\infty} r_{j}^{s}: E\right.$ is covered by $\bigcup_{j=1}^{\infty} B_{j}, B_{j}$ a ball of radius $\left.r_{j} \leq \delta\right\}$.

Clearly, $\mathcal{H}_{\delta}^{s}(E) \leq \mathcal{H}_{\delta^{\prime}}^{s}(E)$ for $\delta^{\prime} \leq \delta$, so the limit $\mathcal{H}^{s}(E)=\lim _{\delta \rightarrow 0} \mathcal{H}_{\delta}^{s}(E)$ exists in $[0, \infty]$ and is called the s-dimensional Hausdorff measure of $E$. The important property is that there exists a critical $\gamma \geq 0$, the so-called Hausdorff dimension of $E$, such that $\mathcal{H}^{s}(E)=\infty$ for all $s<\gamma$ and $\mathcal{H}^{s}(E)=$ 0 for all $s>\gamma$, and the value of $\mathcal{H}^{\gamma}(E)$, if in $(0, \infty)$, is not important. 
This notion of dimension especially applies in the category of $\mathcal{C}^{1}$ manifolds. Let $M$ and $N$ be connected real Riemannian manifolds, of class $\mathcal{C}^{1}$, $\operatorname{dim} M=d \geq 1$ and let $E \subset M$ be closed.

5.7. Proposition ([1], pp. 346-352). (i) $\mathcal{H}^{0}(E)=\operatorname{Card}(E)$;

(ii) $\mathcal{H}^{d}$ coincides with the outer Lebesgue measure on $M$;

(iii) if $\mathcal{H}^{d-1}(E)=0$, then $M \backslash E$ is locally connected;

(iv) let $\pi: M \rightarrow N$ be a $\mathcal{C}^{1}$-smooth map and let $E \subset M$ be such that $\mathcal{H}^{s}(E)=0$ for some $s \geq e=\operatorname{dim} N$. Then $\mathcal{H}^{s-e}\left(E \cap \pi^{-1}(y)\right)=0$ for $d \lambda_{N}$-almost all $y \in N$.

Properties (i), (iii) and (iv) are naturally involved in the proof of the following.

5.8. Lemma. Let $E \subset M_{1}^{-}$be a closed set with $\mathcal{H}_{\mathrm{loc}}^{d-2}(E)=0$. Then for all small $(t, \tau, a, \varrho, p)$, each disc with $A_{t, \tau, a, \varrho, p}(b \Delta) \cap E=\emptyset$ is analytically isotopic to a point in $M \backslash E$.

Proof. During this proof $t, \tau, a$ and $w_{1}^{0}$ are fixed. Then there exist $0<\varrho_{1}, I_{\varrho_{1}}=\left(0, \varrho_{1}\right)$, a neighborhood $\mathcal{V}^{*}$ of 0 in $\mathbb{C}_{w^{*}}^{m-1}, w^{*}=\left(w_{2}, \ldots, w_{m}\right)$ and a neighborhood $\Upsilon$ of 0 in $\mathbb{R}^{n}$ such that the mapping (note that $p$ is parameterized by $\left.\left(w_{1}^{0}, w^{0 *}, y^{0}\right)\right)$

$$
\mathcal{S} \times b \Delta=I_{\varrho_{1}} \times \mathcal{V}^{*} \times b \Delta \ni\left(\varrho, w^{0 *}, y^{0}, \zeta\right) \mapsto A_{t, \tau, a, \varrho, p}(\zeta) \in M
$$

is an embedding. Indeed, this follows by differentiating Bishop's equation, noting first that $\partial Y_{0,0,0,0,0} / \partial y^{0}=\mathrm{Id}, \partial W_{0,0,0,0} / \partial w^{0 *}=\mathrm{Id}$, that $I_{\varrho_{1}} \times b \Delta \ni$ $(\varrho, \zeta) \mapsto \varrho_{1}-\varrho \zeta \in \mathbb{C}$ is an embedding and recognizing that $A_{t, \tau, a, \varrho, p}(\zeta)$ is $\mathcal{C}^{2, \beta}$ with respect to all variables. This exhibits a foliation of an open set in $M$ by $\mathcal{C}^{2, \beta}$ real $\operatorname{discs} \mathcal{D}_{t, \tau, a, p}=\mathcal{D}_{t, \tau, a, w_{1}^{0}, s}, s=\left(w^{0 *}, y^{0}\right)$, where

$$
\mathcal{D}_{t, \tau, a, w_{1}^{0}, s}=\left\{A_{t, \tau, a, \varrho, p}(\zeta) \in M: 0 \leq \varrho<\varrho_{2}, \zeta \in b \Delta\right\} .
$$

Now, since $\mathcal{H}^{d-2}(E)=0$, the set

$$
\mathcal{S} \backslash \mathcal{S}_{E, t, \tau, a, w_{1}^{0}}=\left\{s \in \mathcal{S}: \mathcal{H}^{0}\left(\mathcal{D}_{t, \tau, a, w_{1}^{0}, s} \cap E\right)=0\right\}
$$

is a full measure $(d-2)$-dimensional subset of $\mathcal{S}=I_{\varrho_{1}} \times \mathcal{V}^{*} \times \mathcal{Y} \simeq \mathbb{R}^{d-2}$, by Proposition 5.7(iv). This shows that $\mathcal{D}_{t, \tau, a, w_{1}^{0}, s} \cap E=\emptyset$ for $d \lambda_{\mathcal{S}}$-almost $s=\left(w^{0 *}, y^{0}\right) \in \mathcal{S}$, where $t, \tau, a$ and $w_{1}^{0}$ are fixed. Clearly, the mapping

$$
I_{\varrho_{2}} \cup\{0\} \times \bar{\Delta} \ni(\varrho, \zeta) \mapsto A_{t, \tau, a, \varrho, p}(\zeta) \in \mathbb{C}^{m+n}
$$

yields an analytic isotopy of the analytic $\operatorname{discs} A_{t, \tau, a, \varrho, p}(\zeta)$ for all $0<\varrho \leq$ $\varrho_{1}$, provided $s \in \mathcal{S} \backslash \mathcal{S}_{E, t, \tau, a, w_{1}^{0}}$. It remains to show that discs such that $A_{t, \tau, a, \varrho, p}(b \Delta) \cap E=\emptyset$ but

$$
\mathcal{D}_{t, \tau, a, w_{1}^{0}, s} \cap E \neq \emptyset
$$

are also analytically isotopic to a point in $M \backslash E$. But $\mathcal{H}^{d-2}\left(\mathcal{S}_{E, t, \tau, a, w_{1}^{0}}\right)=0$. Therefore, it suffices to shift slightly the parameter $s$ of $A_{t, \tau, a, \varrho, w_{1}^{0}, s}$ to a 
nearby parameter $s^{\prime}$, which makes $A_{t, \tau, a, \varrho, w_{1}^{0}, s}$ and $A_{t, \tau, a, \varrho, w_{1}^{0}, s^{\prime}}$ isotopic to each other, so that $\mathcal{D}_{t, \tau, a, w_{1}^{0}, s^{\prime}} \cap E=\emptyset$.

The proof of Lemma 5.8 is complete.

STEP 5: Holomorphic extension. Let $v_{00} \in T_{0} M_{1}$ with $v_{00} \notin T_{0}^{c} M$ and $\left|v_{0}-v_{00}\right|<\varepsilon$, and let $C$ be an $n$-dimensional proper linear cone in the $(2 m+n)$-dimensional space $T_{0} M$ which is contained in $\Gamma_{1}$ such that $v_{00} \in C$, the projection $T_{0} C \rightarrow T_{0} M / T_{0}^{c} M$ is surjective and $\bar{C} \cap T_{0}^{c} M=\{0\}$. Fix $p=p_{1}$ and set first $p=0$. Note that $A_{t, \tau, a, \varrho_{1}, 0}(1)=0$. Let $\mathcal{P}_{C}$ denote the set of parameters

$$
\mathcal{P}_{C}=\left\{(t, \tau, a) \in \mathcal{T} \times I_{\tau_{0}} \times \mathcal{A}: \frac{d}{d \theta} A_{t, \tau, a}(1) \in C\right\},
$$

which is a $\mathcal{C}^{1}$-smooth $(n-1)$-dimensional submanifold of $\mathcal{T} \times I_{\tau_{0}} \times \mathcal{A}$. We choose a nearby piece of a manifold, still denoted by $\mathcal{P}_{C}$, with the same tangent space at 0 which is $\mathcal{C}^{2}$-smooth.

Let $K \subset M$ be a germ of a $\mathcal{C}^{1}$-smooth one-codimensional submanifold of $M$ with $0 \in K$ and $T_{0} K \oplus \mathbb{R} \frac{\partial}{\partial \theta} A(1)=T_{0} M$. Let $\mathcal{K}$ be a small neighborhood of 0 in $K$. We denote by $\Delta_{1}$ a small neighborhood of 1 in $\bar{\Delta}$ and $\Delta_{1} \subset \Delta$ its interior.

One observes that a consequence of the isotopy property 5.2 and of the fact that the mapping

$$
\mathcal{P}_{C} \times\left\{\varrho_{1}\right\} \times \mathcal{K} \times \stackrel{\Delta}{1}_{1} \ni\left(t, \tau, a, \varrho_{1}, p, \zeta\right) \mapsto A_{t, \tau, a, \varrho_{1}, p}(\zeta) \in \mathbb{C}^{m+n} \backslash M
$$

is a smooth embedding is that $\mathcal{H}(\omega)$ extends holomorphically into the open wedge set

$$
\mathcal{W}_{\mathcal{P}_{C}}=\left\{A_{t, \tau, a, \varrho_{1}, p}(\zeta):(t, \tau, a) \in \mathcal{P}_{C}, p \in \mathcal{K}, \zeta \in \AA_{1}\right\}
$$

minus the set

$E_{\mathcal{P}_{C}}$

$=\left\{A_{t, \tau, a, \varrho_{1}, p}(\zeta):(t, \tau, a) \in \mathcal{P}_{C}, p \in \mathcal{K} \cap \Phi, A_{t, \tau, a, \varrho_{1}, p}(b \Delta) \cap E \neq \emptyset, \zeta \in \stackrel{\Delta}{1}_{1}\right\}$.

Indeed, since the mapping remains injective on $\mathcal{P}_{C} \times\left\{\varrho_{1}\right\} \times \mathcal{K}$ minus the set $\Phi_{E} \subset \mathcal{P}_{C} \times\left\{\varrho_{1}\right\} \times \mathcal{K}$ of $\left(t, \tau, a, \varrho_{1}, \zeta\right)$ such that the boundary disc meets $E$ at one or more points, we can set unambiguously

$$
F(z):=\frac{1}{2 i \pi} \int_{b \Delta} \frac{f \circ A_{t, \tau, a, p}(\eta)}{\eta-\zeta} d \eta
$$

as a value at points $z=A_{t, \tau, a, \varrho_{1}, p}(\zeta)$ for an extension of $\left.f\right|_{M \backslash E}$, for such $\left(t, \tau, a, \varrho_{1}, p\right) \in \mathcal{P}_{C} \times\left\{\varrho_{1}\right\} \times \mathcal{K} \backslash \Phi_{E}$. Since $f$ extends holomorphically to the interior of these discs, we get a continuous extension $F$ on each $A_{t, \tau, a, \varrho_{1}, p}\left(\Delta_{1}\right)$, $\left(t, \tau, a, \varrho_{1}, p\right) \in \mathcal{P}_{C} \times\left\{\varrho_{1}\right\} \times \mathcal{K} \backslash \Phi_{E}$. Thus, the extension $F$ of $\left.f\right|_{M \backslash E}$ also becomes continuous on 


$$
\left(\mathcal{W}_{\mathcal{P}_{C}} \backslash \mathcal{E}_{\Phi_{E}}\right) \cup(M \backslash E),
$$

where $\mathcal{E}_{\Phi_{E}}$ is the proper closed subset of $\mathcal{W}_{\mathcal{P}_{C}}$ defined by

$$
\mathcal{E}_{\Phi_{E}}=\left\{A_{t, \tau, a, \varrho_{1}, p}(\zeta):\left(t, \tau, a, \varrho_{1}, p\right) \in \Phi_{E}, \zeta \in \stackrel{\Delta}{1}_{1}\right\} .
$$

Since $\left.f\right|_{M \backslash E}$ extends analytically to a neighborhood of $A_{t, \tau, a, \varrho_{1}, p}(\bar{\Delta}), F$ is holomorphic in $\left(\mathcal{W}_{\mathcal{P}_{C}} \backslash \mathcal{E}_{\Phi_{E}}\right)$. Indeed, fix a point $\left(\widetilde{t}, \widetilde{\tau}, \widetilde{a}, \varrho_{1}, \widetilde{p}_{0}\right) \in \mathcal{P}_{C} \times$ $\left\{\varrho_{1}\right\} \times\left(\mathcal{K} \backslash \Phi_{E}\right)$ and let $\widetilde{\mathcal{P}} \times\left\{\varrho_{1}\right\} \times \widetilde{\mathcal{K}}$ be a neighborhood of $\left(\widetilde{t}, \widetilde{\tau}, \widetilde{a}, \varrho_{1}, \widetilde{p}_{0}\right)$ in $\mathcal{P}_{C} \times\left\{\varrho_{1}\right\} \times\left(\mathcal{K} \backslash \Phi_{E}\right)$ such that for each $\left(t, \tau, a, \varrho_{1}, p\right) \in \widetilde{\mathcal{P}} \times\left\{\varrho_{1}\right\} \times \widetilde{\mathcal{K}}$, $A_{t, \tau, a, \varrho_{1}, p}(\bar{\Delta})$ is contained in some neighborhood $\widetilde{\omega}$ of $A_{\widetilde{t}, \widetilde{\tau}, \varrho_{1}, \widetilde{a}, \widetilde{p}_{0}}(\bar{\Delta})$ in $\mathbb{C}^{m+n}$ such that there exists a holomorphic function $\widetilde{f} \in \mathcal{H}(\widetilde{\omega})$ equal to $f$ near $A_{\widetilde{t}, \widetilde{\tau}, \widetilde{a}, \varrho_{1}, \widetilde{p}_{0}}(b \Delta)$. Let $\widetilde{\zeta} \in \stackrel{\Delta}{1}_{1}$ and $\widetilde{z}=A_{\widetilde{t}, \widetilde{\tau}, \widetilde{a}, \varrho_{1}, \widetilde{p}_{0}}(\widetilde{\zeta})$.

To check that the previously defined function $F$ is holomorphic in a neighborhood of $\widetilde{z}$, we notice that for $z=A_{t, \tau, a, p}(\zeta),\left(t, \tau, a, \varrho_{1}, p\right) \in \widetilde{\mathcal{P}} \times$ $\left\{\varrho_{1}\right\} \times \widetilde{\mathcal{K}}, \zeta$ in some neighborhood $\widetilde{\Delta}_{1}$ of $\widetilde{\zeta}$ in $\tilde{\Delta}_{1}, \widetilde{f}(z)$ is given by the Cauchy integral formula

$$
\widetilde{f}(z)=\frac{1}{2 i \pi} \int_{b \Delta} \frac{\tilde{f} \circ A_{t, \tau, a, \varrho_{1}, p}(\eta)}{\eta-\zeta} d \eta=\frac{1}{2 i \pi} \int_{b \Delta} \frac{f \circ A_{t, \tau, a, \varrho_{1}, p}(\eta)}{\eta-\zeta} d \eta=F(z) .
$$

As a consequence, $\widetilde{f}(z)=F(z)$ for $z$ in a small neighborhood of $\widetilde{z}$ in $\mathbb{C}^{m+n}$, since the mapping $(t, \tau, a, p, \zeta) \mapsto A_{t, \tau, a, \varrho_{1}, p}(\zeta)$ from $\widetilde{\mathcal{P}} \times \widetilde{\mathcal{K}} \times \widetilde{\Delta}_{1}$ to $\mathbb{C}^{m+n}$ has $\operatorname{rank} 2 n$ at $\left(\tilde{t}, \widetilde{\tau}, \widetilde{a}, \widetilde{p}_{0}, \widetilde{\zeta}\right)$.

This proves that $F$ is holomorphic in $\mathcal{W}_{\mathcal{P}_{C}} \backslash \mathcal{E}_{\Phi_{E}}$.

By shrinking $\omega$ near 0, which does not modify the possible disc deformations, we can ensure that $\omega \cap \mathcal{W}_{\mathcal{P}_{C}}$ is connected, since $\bar{C} \cap T_{0}^{c} M=\{0\}$. By Lemma 5.9 below the same is true for $\omega \cap \mathcal{W}_{\mathcal{P}_{C}} \backslash \mathcal{E}_{\Phi_{E}}$. Indeed, the fact that $\mathcal{E}_{\Phi_{E}}$ is of zero $(2 m+2 n-1)$-dimensional Hausdorff measure implies the connectedness. Therefore $f \in \mathcal{H}(\omega)$ and $F \in \mathcal{H}\left(\mathcal{W}_{\mathcal{P}_{C}} \backslash \mathcal{E}_{\Phi_{E}}\right)$ stick together into a single holomorphic function in $\omega \cup\left(\mathcal{W}_{\mathcal{P}_{C}} \backslash \mathcal{E}_{\Phi_{E}}\right)$, since both are continuous up to $M \backslash E$, which is a uniqueness set, and coincide there.

5.9. Lemma. The set $\mathcal{E}_{\Phi_{E}}$ is a union of complex curves and

$$
\mathcal{H}^{2 m+2 n-1}\left(\mathcal{E}_{\Phi_{E}}\right)=0 .
$$

Pro of. Namely, near the origin,

$$
\mathcal{E}_{\Phi_{E}}=\bigcup_{\left(t, \tau, a, \varrho_{1}, p\right) \in \Phi_{E}} A_{t, \tau, a, \varrho_{1}, p}\left(\stackrel{\circ}{1}_{1}\right) .
$$

Fix $t \in C, \tau$ and $a$, and consider the sets

$$
M_{t, \tau, a}=\left\{A_{t, \tau, a, \varrho_{1}, p}(\zeta): p \in \mathcal{K}, \zeta \in \AA_{1}\right\},
$$

depending on $t$. Then $\mathcal{W}_{\mathcal{P}_{C}}$ is foliated near 0 by the manifolds $M_{t, \tau, a}$, $\operatorname{dim} M_{t, \tau, a}=1+\operatorname{dim} M$, that contain $M$ in their boundaries near 0 and 
$M$ is foliated by the $\operatorname{arcs} A_{t, \tau, a, \varrho_{1}, p}\left(\Delta_{1} \cap b \Delta\right)$. Notice that $A_{t, \tau, a, \varrho_{1}, p}(b \Delta) \cap$ $E=A_{t, \tau, a \varrho_{1}, p}\left(b \Delta \cap \Delta_{1}\right) \cap E$ by the choice of $A=A_{0}$ with $A(1) \in M_{1}$, $A(b \Delta \backslash\{1\}) \subset M_{1}^{+} \backslash M_{1}$, by $E \subset M_{1}^{-}$and by continuity. A direct application of Proposition 5.7(iv) entails that, for all fixed $(t, \tau, a)$, the set

$$
\mathcal{K}_{E, t, \tau, a}=\left\{p \in \mathcal{K}: A_{t, \tau, a, \varrho_{1}, p}\left(b \Delta \cap \Delta_{1}\right) \cap E \neq \emptyset\right\} \subset \mathcal{K},
$$

contained in the $(d-1)$-dimensional manifold $\mathcal{K}$, satisfies

$$
\mathcal{H}^{d-2}\left(\mathcal{K}_{E, t, \tau, a}\right)=0 .
$$

Therefore, $\mathcal{H}^{d}\left(M_{E, t, \tau, a}\right)=0$ too in $M_{t, \tau, a}$ and since $\mathcal{W}_{\mathcal{P}_{C}}$ is regularly foliated by the $M_{t, \tau, a}$,

$$
\mathcal{H}^{2 m+2 n-1}\left(\mathcal{E}_{\Phi_{E}}\right)=\mathcal{H}^{2 m+2 n-1}\left(\bigcup_{(t, \tau, a) \in \mathcal{P}_{C}} M_{E, t, \tau, a}\right)=0 .
$$

The proof of Lemma 5.9 is complete.

Remark. By Proposition 5.7(iii), the set $\mathcal{K} \backslash \mathcal{K}_{E, t, \tau, a}$ is connected. This provides another proof of the isotopy property of Lemma 5.8.

A closed set $\mathcal{E}$ contained in an open set $\mathcal{W} \subset \mathbb{C}^{m+n}$ with $\mathcal{H}^{2 m+2 n-1}(\mathcal{E})$ $=0$ not being automatically removable, we must study the structure of $\mathcal{E}_{\Phi_{E}}$ with respect to the local complex structure of $\mathcal{W}$.

The hypersurface case. Let us first give a proof for removing $\mathcal{E}_{\Phi_{E}}$ in the case $m=n=1$. To begin the variations on this theme, recall that $\mathcal{E}_{\Phi_{E}}$ would be removable if $\mathcal{H}^{2 m+2 n-2}\left(\mathcal{E}_{\Phi_{E}}\right)=0$, which completes the proof of Theorem 5.1(ii).

Theorem (see [1]). Let $\mathcal{E}$ be a closed subset of an open set $\mathcal{U} \subset \mathbb{C}^{m+n}$, $m+n \geq 1$, with $\mathcal{H}^{2 m+2 n-2}(\mathcal{E})=0$. Then, for every function $f \in \mathcal{H}(\mathcal{U} \backslash \mathcal{E})$, there exists a function $F \in \mathcal{H}(\mathcal{U})$ such that $F=f$ in $\mathcal{U} \backslash \mathcal{E}$.

Proof. We can, by localization, assume that $\mathcal{U}=P$ is the unit polydisc $\Delta^{m+n}$ and that $0 \in \mathcal{E}$. Let $G(k, m+n), 0 \leq k \leq m+n$, denote the grassmannian of $k$-dimensional complex planes passing through the origin in $\mathbb{C}^{m+n}$.

Proposition ([1]). Let $\mathcal{E}$ be a closed subset in $\Delta^{m+n}$ such that $\mathcal{H}^{2 k+1}(\mathcal{E})$ $=0$ for some integer $k<m+n$. Then $\mathcal{H}^{1}(\mathcal{E} \cap L)=0$ for almost every plane $L \in G(m+n-k, m+n)$.

Choose therefore a complex line $L$ through $0 \in \mathcal{E}$ such that $\mathcal{H}^{1}(L \cap$ $\left.\Delta^{m+n} \cap \mathcal{E}\right)=0$ and an orthogonal $(m+n-1)$-dimensional complex space $H$, $H \oplus L=\mathbb{C}^{m+n}$. By Proposition 5.7(iv), for almost all $h \in H,\{h\} \times L \cap \mathcal{E}=\emptyset$. Write $\Delta_{L}=L \cap \Delta^{m+n}$, choose a point $p \in \Delta_{L} \cap \mathcal{E}$ (e.g. 0 ) and draw a small complex disc $\Delta_{L}\left(p, r_{0}\right)$ of radius $r_{0}>0$ and center $p$ with $\overline{\Delta_{L}\left(p, r_{0}\right)} \subset \Delta_{L}$. For almost all $r<r_{0}, b \Delta_{L}(p, r) \cap \mathcal{E}=\emptyset$, still thanks to Proposition 5.7(iv). 
Furthermore, $\overline{\Delta_{L}(p, r)}+h \subset \Delta^{m+n} \backslash \mathcal{E}$ for arbitrarily small $h \in H$. Therefore, such a disc yields removability of $p$ for $\mathcal{H}\left(\Delta^{m+n} \backslash \mathcal{E}\right)$ along an obvious isotopy.

The proof is complete.

Proposition. Let $J$ and $K$ be closed subsets of $\Delta$ with $\mathcal{H}^{1}(J)=\mathcal{H}^{1}(K)$ $=0$ and set $\mathcal{E}=(J \times \Delta) \cap(\Delta \times K) \subset \Delta^{2}$. Then $\mathcal{E}$ is removable for $\mathcal{H}\left(\Delta^{2} \backslash \mathcal{E}\right)$.

Proof. Fix $f \in \mathcal{H}\left(\Delta^{2} \backslash \mathcal{E}\right)$. For all $\zeta_{2} \in \Delta \backslash K$, one has $\Delta \times\left\{\zeta_{2}\right\} \subset \Delta^{2} \backslash \mathcal{E}$. For $\zeta_{2} \in K$,

$$
\left(\Delta \times\left\{\zeta_{2}\right\}\right) \cap \mathcal{E}=\left(\Delta \times\left\{\zeta_{2}\right\}\right) \cap(J \times \Delta)=J \times\left\{\zeta_{2}\right\},
$$

so $\mathcal{H}^{1}\left(\left(\Delta \times\left\{\zeta_{2}\right\}\right) \cap E\right)=0$ and $f$ is holomorphic near each point $\left(\zeta_{1}, \zeta_{2}\right) \in$ $\Delta \times\left\{\zeta_{2}\right\} \backslash \mathcal{E}$. There exists $\lambda_{2} \in \mathbb{C}$ arbitrarily small with $\Delta \times\left\{\zeta_{2}+\lambda_{2}\right\} \subset$ $\Delta^{2} \backslash \mathcal{E}$. Applying Proposition 5.7(iv), we have $\mathcal{H}^{0}\left(r b \Delta \times\left\{\zeta_{2}\right\} \cap \mathcal{E}\right)=0$ for almost all $0<r<1$. In other words, $r b \Delta \cap \mathcal{E}=\emptyset$. For such $r$, the continuity principle along the family $(s, \zeta) \mapsto\left(r \zeta,(1-s) \lambda_{2}+\zeta_{2}\right), 0 \leq s \leq 1$, yields an extension of $f$ in a neighborhood $\mathcal{V}\left(r \bar{\Delta} \times\left\{\zeta_{2}\right\}\right) \subset \Delta^{2}$. Notice that $\mathcal{V}\left(r \bar{\Delta} \times\left\{\zeta_{2}\right\}\right) \cap \Delta^{2} \backslash \mathcal{E}$ is connected, since $\Delta^{2} \backslash(\Delta \times K)$ and $\Delta^{2} \backslash(J \times \Delta)$ are connected, by Proposition 5.7(iii), so uniqueness is guaranteed.

The proof is complete.

REMARK. The argument above relies upon two facts: 1) almost all complex discs lay in $\left.\Delta^{2} \backslash \mathcal{E} ; 2\right)$ every disc touching $\mathcal{E}$ satisfies $\mathcal{H}^{1}(\Delta \cap \mathcal{E})=0$.

These preliminaries provide us with a proof of Theorem 5.1 in the case where $M \subset \mathbb{C}^{2}$ is a $\mathcal{C}^{2, \alpha}$ hypersurface. Indeed, here $\mathcal{A}=\emptyset$ and we can choose small $\left(t_{1}, \tau_{1}\right)$ and $\left(t_{2}, \tau_{2}\right)$ so that $v_{1}$ and $v_{2}$ are linearly independent, $v_{1}=(\partial / \partial \theta) A_{t_{1}, \tau_{1}, \varrho_{1}, 0}(1)$ and $v_{2}=(\partial / \partial \theta) A_{t_{2}, \tau_{2}, \varrho_{1}, 0}(1)$ so that $v_{1}$ and $v_{2}$ point to the same side of $T_{0}^{c} M$ relative to $T_{0} M$ and $v_{1}, v_{2} \notin T_{0}^{c} M$. This is clearly possible, by Lemma 5.6. Denote these two families simply by $A_{1, p}(\zeta)$ and $A_{2, p}(\zeta)$. Then, since the normal bundle to $M$ is of rank one and since $J v_{1}, J v_{2}$ point to the same side of $M$ in $\mathbb{C}^{2}$, the following two wedges (same one-sided neighborhood) with edge $M$ at 0 :

$$
\mathcal{W}_{1}=\left\{A_{1, p}\left(\AA_{1}\right): p \in \mathcal{K}\right\}, \quad \mathcal{W}_{2}=\left\{A_{2, p}\left(\AA_{1}\right): p \in \mathcal{K}\right\}
$$

contain a side $\mathcal{W}$ of boundary $M$ at 0 . As above, one can construct two holomorphic extensions $F_{1}$ and $F_{2}$ of $f$ to $\mathcal{W}_{1} \backslash \mathcal{E}_{1}$ and $\mathcal{W}_{2} \backslash \mathcal{E}_{2}$ respectively, where $\mathcal{E}_{1}$ and $\mathcal{E}_{2}$ denote the sets corresponding to the discs touching $E$ :

$$
\mathcal{E}_{1}=\left\{A_{1, p}(\zeta): p \in \mathcal{K}, \zeta \in \stackrel{\circ}{\Delta}_{1}, A_{1, p}\left(b \Delta \cap \Delta_{1}\right) \cap E \neq \emptyset\right\}
$$

and similarly for $\mathcal{E}_{2}$. By Proposition 5.7(iv), the one-dimensional Hausdorff measure of $\Phi_{j}=\left\{p \in \mathcal{K}: A_{j, p}(b \Delta) \cap E \neq \emptyset\right\}, j=1,2$, is zero. Indeed, recall that $A(b \Delta) \cap E=A\left(b \Delta \cap \Delta_{1}\right) \cap E$ for such discs with $p$ very close to 0 (in comparison with the size of $\left.\Delta_{1}\right)$ and that $\left\{A_{j, p}\left(b \Delta \cap \Delta_{1}\right): p \in \mathcal{K}\right\}$ foliate a neighborhood of 0 in $M$. 
Let $z \in \mathcal{W} \subset \mathcal{W}_{1} \cap \mathcal{W}_{2}$ be such that $z \in \mathcal{E}_{1}$ or $z \in \mathcal{E}_{2}$, say $z \in \mathcal{E}_{1}$, $z=A_{1, p_{1, z}}\left(\zeta_{1, z}\right)$ and $z=A_{2, p_{2, z}}\left(\zeta_{2, z}\right)$. Notice first that there are points $p \in \mathcal{K}$ arbitrarily close to $p_{1, z}$ such that $A_{1, p}(b \Delta) \subset M \backslash E$. Second, since $A_{1, p_{1, z}}$ and $A_{2, p_{2, z}}$ are transversal in $\mathbb{C}^{2}$ at $z$ (by the choice of $\left(v_{1}, v_{2}\right)$ ), for all points $p$ varying in a neighborhood $\mathcal{V}\left(p_{1, z}\right) \subset \mathcal{K}$, the discs $A_{1, p_{1, z}}$ and $A_{2, p}$ intersect transversally in a single point $z(p)$ such that $z\left(p_{2, z}\right)=z$ and $\mathcal{V}\left(p_{1}\right) \ni p \mapsto z(p) \in A_{2, p}\left(\AA_{1}\right)$ is a local $\mathcal{C}^{1}$ diffeomorphism. But only for $p \in \mathcal{E}_{2}$ is the disc $A_{2, p}$ not analytically isotopic to a point in $M \backslash E$ and $\mathcal{H}^{3}\left(\mathcal{E}_{2}\right)=0$. Thus, this shows that $F$ is already holomorphic at each point of $\mathcal{V}(z) \cap A_{1, p_{1, z}}\left(\stackrel{\circ}{1}_{1}\right)$ outside a thin closed subset $e_{1} \subset A_{1, p_{1, z}}\left(\stackrel{\circ}{1}_{1}\right)$ with $\mathcal{H}^{1}\left(e_{1}\right)=0$. Now, there exists a small circle contained in this disc not meeting $e_{1}$ and the isotopy used in the proof above can be applied once again in this analogous context to prove that $z$ is removable for $F \in$ $\mathcal{H}\left(\mathcal{W} \backslash\left(\mathcal{E}_{1} \cap \mathcal{E}_{2}\right)\right)$.

REMARK. A general proof in the hypersurface case (i.e. for $m \geq 1$ ) follows along the same lines as above or by reduction to $\mathbb{C}^{2}$ by slicing and using a separate analyticity theorem like, for example, Shiffman's theorem below (see Chirka and Stout [2], Section 4, for related reductions). This completes the proof of Theorem 5.1(i).

End of proof of Theorem 5.1. By construction, there exists $F \in \mathcal{H}(\omega \cup$ $\left.\left(\mathcal{W}_{\mathcal{P}_{C}} \backslash \mathcal{E}_{\Phi_{E}}\right)\right)$ extending $f$. Let $A$ be a disc in the family generating $\mathcal{W}_{\mathcal{P}_{C}}$ and assume that $A\left(b \Delta \cap \Delta_{1}\right) \cap E \neq \emptyset$. We shall remove $A\left(\stackrel{\Delta}{1}_{1}\right)$. Notice that, by construction, $A\left(b \Delta \cap \Delta_{1}\right) \not \subset E$.

There always exists a point $p=A\left(\zeta_{p}\right), \zeta_{p} \in \AA_{1}$, such that $p \in b \omega \cap \mathcal{E}_{\Phi_{E}}$. For simplicity, write $\mathcal{W}=\mathcal{W}_{\mathcal{P}_{C}}$ and $\mathcal{E}=\mathcal{W}_{\mathcal{P}_{C}} \backslash\left(\omega \cup\left(\mathcal{W}_{\mathcal{P}_{C}} \backslash \mathcal{E}_{\Phi_{E}}\right)\right), F \in$ $\mathcal{H}(\mathcal{W} \backslash \mathcal{E})$

By imitating the first step reduction, we can assume (see the explanation below) that, after perhaps changing $p$, there is a germ of a one-codimensional manifold $M_{1}$ such that $p \in M_{1}$ and the remaining part of $\mathcal{E}$ to be removed is contained in a half-space $M_{1}^{-}$.

Indeed, a neighborhood $\mathcal{U}$ of $p$ in $\mathcal{W}$ is foliated by discs of the family generating the wedge $\mathcal{W}\left(=\mathcal{W}_{\mathcal{P}_{C}}\right)$ and these analytic discs are integral real surfaces of a subbundle, say $K$, of $T \mathcal{W}$, which they span. In this neighborhood, one half of each disc lies in $\omega \cap \mathcal{U}$, the other half is outside $\omega$ and all discs are transversal to $b \omega$ (assuming, from the beginning, that $b \omega \backslash M$ is smooth after shrinking $\omega)$.

Furthermore, since $A\left(b \Delta \cap \Delta_{1}\right) \not \subset E$ for each disc of the family (hence $\left.A\left(\AA_{1}\right) \cap \omega \neq \emptyset\right)$, setting $\mathcal{E}=\mathcal{W}_{\mathcal{P}_{C}} \backslash\left(\omega \cup\left(\mathcal{W}_{\mathcal{P}_{C}} \backslash \mathcal{E}_{\Phi_{E}}\right)\right)$, one sees that $\mathcal{W}$ and $\mathcal{W} \backslash \mathcal{E}$ are $K$-minimal, i.e. both are a single $K$-orbit.

This is a key geometric fact (cf. [11], [15], [17], [16] and 5.1 here). 
Then after introducing a set $\mathcal{A}$ as in Step 1, and after making use of the differential geometric lemma quoted in Step 1, we remove a point $p=$ $A\left(\zeta_{p}\right) \in b \omega$ and the other ones are removed similarly.

To complete the proof of Theorem 5.1(iii), recall that if $M$ is realanalytic, it is known by works of Bloom-Graham or Baouendi-Rothschild that there exists a real-analytically parameterized family of analytic discs attached to $M$ as $A_{t, \tau, a, \varrho, p}$ filling a wedge at the base point so that the foliation of $\mathcal{W}$ by pieces of $A\left(\dot{\Delta}_{1}\right)$ is a real-analytic foliation. Furthermore, in contrast to the globally minimal case, it is superfluous to deform $M$ step by step after removing points of $E$, since 1) $M$ is already minimal at every point and 2) the isotopy Lemma 5.8 holds without assuming that $E$ is contained in a half-space $M_{1}^{-}$.

5.10. TheOREM. Let $\mathcal{U} \subset \mathbb{C}^{m+n}$ be a domain (connected) equipped with a $\mathcal{C}^{\omega}$ foliation by complex-analytic curves and let $\mathcal{E} \subset \mathcal{U}$ be a closed subset which is a union of leaves, with $\mathcal{H}^{2 m+2 n-1}(\mathcal{E})=0$. Then a function $F \in \mathcal{H}(\mathcal{U} \backslash \mathcal{E})$ extends holomorphically to a neighborhood of a whole leaf $A$ whenever $F$ extends holomorphically through a single point of $A$.

REMARK. When $m=n=1$, we provide a proof that $\mathcal{E}$ is removable for a $\mathcal{C}^{2, \alpha}$ foliation in Theorem 5.13 below.

Proof. After the above reduction, the geometric assumption is: there exists a neighborhood $\mathcal{U}$ of $p$ which is foliated by complex-analytic curves, $\mathcal{U}=\bigcup_{\theta \in D} A_{\theta}, D \subset \mathbb{R}^{2 m+2 n-2}$ a small open set, there exists a closed set $\mathcal{G} \subset D$ with $\mathcal{H}^{2 m+2 n-3}(\mathcal{G})=0$ (corresponding in the proof of Theorem 5.1 to the discs attached to $M$ which meet $E$ ) and the set $\mathcal{E}$ to be removed (in $\mathcal{U} \equiv \mathcal{W}$ near $p$ ) is a union of half discs, $\mathcal{E}=\bigcup_{\theta \in \mathcal{G}} A_{\theta}^{-}$, where $A_{\theta}^{-}=A_{\theta} \cap M_{1}^{-}$.

5.11. Proposition. Let $\mathcal{U} \subset \mathbb{C}^{m+n}$ be a small open set $\mathcal{C}^{\omega}$-foliated by complex curves $A_{\theta}, \mathcal{U}=\bigcup_{\theta \in D} A_{\theta}, 0 \in \mathcal{U}, D \subset \mathbb{R}^{2 m+2 n-2}$ open, $0 \in D$, let $\mathcal{G} \subset D$ be a closed set with $\mathcal{H}^{2 m+2 n-3}(\mathcal{G})=0$, let $M_{1}$ be a $\mathcal{C}^{1}$ hypersurface through 0 in $\mathcal{U}$ with $T_{0} M_{1}+T_{0} A_{0}=T_{0} \mathbb{C}^{m+n}$ and set $\mathcal{E}=\left(\bigcup_{\theta \in \mathcal{G}} A_{\theta}\right) \cap M_{1}^{-}$. Then there exists a neighborhood $\mathcal{V}$ of 0 such that for every function $f \in$ $\mathcal{H}(\mathcal{U} \backslash \mathcal{E})$, there exists a function $f \in \mathcal{H}(\mathcal{V})$ with $F=f$ in $\mathcal{V} \backslash \mathcal{E}$.

Proof. It is not true that the foliation of $\mathcal{W}$ by discs of the foliation is a complex-analytic foliation, i.e. locally equivalent to $\mathbb{C} \times \mathbb{C}^{m+n-1}$ after a biholomorphism (neither in 5.10 nor in 5.11).

Nevertheless, let us first investigate geometrically this case.

The case of a holomorphic foliation. Here, the geometric situation is that there exists a smooth hypersurface $M_{1}$ through 0 and a closed set $\mathcal{G} \subset \Delta^{m+n-1}$ with $\mathcal{H}^{2 m+2 n-3}(\mathcal{G})=0$ such that $\mathcal{E}=M_{1}^{-} \cap(\Delta \times \mathcal{G})$ near 0 . Indeed, one just straightens the holomorphic foliation. 
Notice that by Proposition 5.7(iv), for almost all two-dimensional affine complex planes $L \equiv \mathbb{C} \times \mathbb{C}, L \cap(\mathbb{C} \times \mathcal{G})=\mathbb{C} \times \mathcal{G}_{L} \subset \mathbb{C} \times \mathbb{C}$ for a closed set $\mathcal{G}_{L} \subset \mathbb{C}$ with $\mathcal{H}^{1}\left(\mathcal{G}_{L}\right)=0$. Hence we are in the following situation (a particular case of Proposition 5.11).

5.12. Lemma. Let $\mathcal{U}$ be a connected open set in $\mathbb{C}_{w, z}^{2}, 0 \in \mathcal{U}$, let $M_{1} \subset \mathcal{U}$ be a closed hypersurface, $0 \in M_{1}, T_{0} M_{1} \oplus \mathbb{R}_{u}=T_{0} \mathbb{C}^{2}, u=\operatorname{Re} w$, and let $\mathcal{E}=\left(\mathbb{C}_{w} \times E\right) \cap \mathcal{U} \cap M_{1}^{-}$be closed, where $E \subset \mathbb{C}_{z}$ is closed and $\mathcal{H}_{\text {loc }}^{1}(E)=0$. Then there exists a neighborhood $\mathcal{V}$ of 0 such that for every function $f \in$ $\mathcal{H}(\mathcal{U} \backslash \mathcal{E})$, there exists a function $F \in \mathcal{H}(\mathcal{V})$ with $F=f$ in $\mathcal{V} \backslash \mathcal{E}$.

Proof. Notice that $\mathcal{H}^{3}(\mathcal{E})=0$. Define $B_{w}(\zeta)=(w, r \zeta),|w|$ small and $r>0$. Since $\mathcal{H}^{1}(E)=0$, for almost all $r>0$ the boundary of the disc $\zeta \mapsto r \zeta$ does not meet $E$. Hence also $B_{w}(b \Delta) \cap \mathcal{E}=\emptyset$ for such $r>0$, because $\mathcal{E} \subset \mathbb{C}_{w} \times E$. Fix such an $r$. Then all $B_{w}$ for different $w$ are analytically isotopic to each other in $\mathcal{U} \backslash \mathcal{E}, B_{0}(0)=0$ and, moreover, $B_{u}$ is analytically isotopic to the point $(u, 0)$ in $\mathcal{U} \backslash \mathcal{E}$ if $u>0$. Therefore 0 is removable.

The proof of Lemma 5.12 is complete.

For general $m+n \geq 2$, the above constructed isotopies lie inside a fixed complex plane $L$, so that the continuity principle in $\mathbb{C}^{m+n}$ applies, giving holomorphic extension at 0 .

REMARK. The $\mathcal{C}^{2, \alpha}\left(\right.$ even $\mathcal{C}^{1}$ ) foliated version of Theorem 5.10 admits a proof in $\mathbb{C}^{2}$ that we give below.

5.13. Theorem. Let $\mathcal{U} \subset \mathbb{C}^{2}$ be a domain equipped with a $\mathcal{C}^{1}$ foliation $\mathcal{F}$ by complex-analytic curves. Further let $\mathcal{E}$ be a closed union of leaves with $\mathcal{H}^{3}(\mathcal{E})=0$. If a function $f \in \mathcal{H}(D \backslash \mathcal{E})$ admits a holomorphic extension to a neighborhood of some point $p \in \mathcal{E}$, then it extends analytically to a neighborhood of the leaf $L$ containing $p$.

Proof. Let $L^{\prime}$ be the set of all points $z \in L$ such that $f$ extends holomorphically to a neighborhood of $z$. As all such extensions obviously fit together, it is enough to show $L^{\prime}=L$.

Assume $L^{\prime} \neq L$. Clearly $L^{\prime}$ is an open subset of $L$ with respect to the leaf topology. Fix a point $q$ on the relative boundary of $L^{\prime}$. In a neighborhood $U$ of $q$ we choose a holomorphic function $z$ with $z(q)=0, d z(q) \neq 0$, such that the curve $\{z=0\}$ intersects the leaf $L$ transversally in $q$. Hence $\mathcal{H}^{1}(\mathcal{E} \cap\{z=0\})=0$ (after contraction of $U$ ), and we may draw a simple closed curve $\gamma_{0} \subset\{z=0\}$ surrounding $q$ and avoiding $\mathcal{E} \cap\{z=0\}$.

If we choose $\gamma_{0}$ in a small neighborhood of $q$, we get a family of contours $\gamma_{\zeta} \subset\{z=\zeta\} \backslash \mathcal{E},|\zeta| \leq \varepsilon$, by moving $\gamma_{0}$ along the foliation. By hypothesis, for an open set of parameters $\zeta$, the restriction of $f$ to $\{z=\zeta\}$ is holomorphic near the closure of the domain $G_{\zeta}$ surrounded by $\gamma_{\zeta}$. As $f$ is holomorphic 
near $\bigcup_{|\zeta| \leq \varepsilon} \gamma_{\zeta}$, it extends by the continuity principle to a neighborhood of $\bigcup_{|\zeta| \leq \varepsilon} \bar{G}_{\zeta}$, contrary to the choice of $q$.

The proof of Theorem 5.13 is complete.

REMARK. However, the reduction to $\mathbb{C}^{2}$ is impossible for a general $\mathcal{C}^{2, \alpha}$ foliation, since there need not be families of complex surfaces foliated by complex curves of the foliation. Therefore, 5.13 in $\mathbb{C}^{2}$ does not provide 5.13 for any $\mathbb{C}^{m+n}$.

The case of a real-analytic foliation. Here, we use a separate analyticity theorem due to Shiffman. A subset $Q$ of a polydisc $\Delta^{m+n-1}$ is said to be a full subset of $\Delta^{m+n-1}$ if $D \cap Q$ is a set of full measure in $D$ for almost every coordinate disc $D \subset \Delta^{m+n-1}$.

Theorem (Shiffman [20]). Let $\Delta^{m+n-1} \Subset \mathbb{C}^{m+n-1}$ be a polydisc and let $Q \subset \Delta^{m+n-1}$ be a full subset of $\Delta^{m+n-1}$. Then a function $F: Q \rightarrow \mathbb{C}$ has a holomorphic extension to $\Delta^{m+n-1}$ if and only if, for almost every coordinate disc $D \subset \Delta^{m+n-1},\left.F\right|_{D \cap Q}$ extends holomorphically to $D$.

First, $p=0$ in coordinates $(w, z) \in \mathbb{C} \times \mathbb{C}^{m+n-1}, w=u+i v$, with $\mathbb{C} \times\{0\}=T_{0} A_{0}$ and $T_{0} M_{1}=\{u=0\}, T_{0} M_{1}^{-}=\{u \leq 0\}$. There are, in the whole grassmannian of affine complex lines passing near 0 in $\mathcal{U}$, the lines $a+h\left(L_{0}\right), a \in\{0\} \times \mathbb{C}^{m+n-1}$ close to 0 , with $h \in \mathrm{GL}(m+n, \mathbb{C})$ close to Id and $L_{0}=\mathbb{C}_{w} \times 0$, which are cut by $M_{1}$ in two pieces. Draw a small enough analytic disc $B(\zeta)=(c(\zeta-1)-b, 0), \zeta \in \Delta$, inside $L_{0}$, with $c>0$ small and fixed throughout, with $b>0, b \ll c$, and define $B_{a, h}(\zeta):=a+h \circ B(\zeta)$ so that $B_{a, h}(\Delta) \subset a+h\left(L_{0}\right)$. Notice that for small $|a| \ll c,\|h-\operatorname{Id}\| \ll c$, then $B_{a, h}(b \Delta) \cap M_{1}^{-} \subset B_{a, h}\left(b \Delta \cap \Delta_{1}\right)$, for fixed $\Delta_{1}=\left\{|\zeta-1| \leq c_{1}\right\} \cap \bar{\Delta}$, with $c_{1}=c / 5$ say. (In other words, the boundaries $B_{a, h}(b \Delta)$ can meet $\mathcal{E}$ only along a fixed part of them.) And notice that for fixed $h, c, \bigcup_{a} B_{a, h}(\Delta)$ is a holomorphic foliation by complex discs with the origin point in its interior. Varying $h$, to apply Shiffman's theorem, it suffices to show that for almost all $a \in \mathbb{C}^{m+n-1}$ close to 0 , a fixed function $F \in \mathcal{H}(\mathcal{U} \backslash \mathcal{E})$ extends holomorphically to $B_{a, h}(\Delta)$.

Clearly, if $c$ is small, then $B_{a, h}(b \Delta) \cap M_{1}^{-} \subset B_{a, h}(b \Delta \cap\{|\zeta-1| \leq 5 b\})$ for all $a, h$, so that $B_{a, h}\left(b \Delta \cap \Delta_{1}\right)$ is much longer than its intersection with $M_{1}^{-}$.

Let $\Sigma_{1}=\bigcup_{a} B_{a, h}\left(b \Delta \cap \Delta_{1}\right)$. The hypersurface $\Sigma_{1}$ is transversal to the real foliation, hence $\mathcal{H}^{2 m+2 n-2}\left(\Sigma_{1} \cap \mathcal{E}\right)=0$. Hence by Proposition 5.7(iv), $B_{a, h}\left(b \Delta \cap \Delta_{1}\right) \cap \mathcal{E}=\emptyset$ for almost all $a$ (by the property $\mathcal{H}^{1}\left(B_{a, h}\left(b \Delta \cap \Delta_{1}\right)\right)=$ $0)$. In other words, $B_{a, h}(b \Delta) \cap \mathcal{E}=\emptyset$ for almost all $a$. To complete the proof of Theorem 5.10, the remaining point is to isotope the boundaries $B_{a, h}(b \Delta)$ analytically to a point inside $\mathcal{U} \backslash \mathcal{E}$.

By the foliation assumption, there exist some real-analytic coordinates $(u, v, x)$ so that $\mathcal{U}=\mathbb{R}_{u, v}^{2} \times \mathbb{R}_{x}^{2 m+2 n-2}$ (here, $(u, v)$ are real coordinates 
in general distinct from $(\operatorname{Re} w, \operatorname{Im} w))$ such that the sets $\mathbb{R}_{u, v}^{2} \times\{$ const $\}$ correspond to discs of the foliation and $\mathcal{E}=M_{1}^{-} \times\left(\mathbb{R}_{u, v}^{2} \times \mathcal{G}\right)$. We can assume that the Jacobian matrix of the change from the holomorphic coordinates $(w, z)$ to $(u, v, x)$ is the identity at the origin (so that $u$ and $v$ are close to $\operatorname{Re} w$ and $\operatorname{Im} w)$.

Define $\gamma_{B}:=B_{a, h}\left(b \Delta \cap \Delta_{1}\right)$ and set

$$
\Sigma_{a, h}=\left\{p+u \in \mathcal{U}: p \in \gamma_{B},-5 b<u<5 b\right\}
$$

in these real-analytic coordinates and come back to the original holomorphic coordinates.

Then $\Sigma_{a, h}$ is a piece of a $\mathcal{C}^{\omega}$-smooth surface near 0 with $\Sigma_{a, h} \cap \mathcal{E}=\emptyset$. However, $\Sigma_{a, h}$ is not immediately seen to be a union of boundaries of analytic discs as in the case of a holomorphic foliation.

Therefore, we shall apply a complexification argument.

From the beginning, we can assume that $b \ll c,|a| \ll c,|a| \approx b$ and also $\|h-\operatorname{Id}\| \ll|a|$, since the set of such $h$ still contains an open set in $\operatorname{GL}(m+n, \mathbb{C})$, which is sufficient to apply separate analyticity locally. Therefore, for all $p \in \Sigma_{a, h}$, the tangent space $T_{p} \Sigma_{a, h}$ (considered as a linear subspace of $\left.T_{0} \mathbb{R}^{2 m+2 n}\right)$ is close to $T_{0} L_{0}=\mathbb{C}_{w} \times\{0\}$. Therefore, $\Sigma_{a, h}$ is a graph over a domain $D_{a, h}$ contained in the $w$-space. Since the transformation from $(w, z)$ to $(u, v, x)$ is close to the identity in the $\mathcal{C}^{1}$ norm and since $\|h-\operatorname{Id}\|$ and $|a|$ are very small, this domain $D_{a, h} \subset \mathbb{C}_{w}$ is approximately the domain

$$
D:=\left\{c(\zeta-1)-b+u_{0} \in \mathbb{C}_{w}: \zeta \in b \Delta,|\zeta-1|<c / 5,-5 b<u_{0}<5 b\right\} .
$$

Anyway, by taking $\Sigma_{a, h}$ a little bit larger, $D_{a, h}$ will contain $D$ for all small $|a|,\|h-\mathrm{Id}\|$.

Hence there exist $\mathcal{C}^{\omega}$ functions $s_{j}: D \rightarrow \mathbb{C}, 1 \leq j \leq m+n-1$, such that $\Sigma_{a, h}$ is given by the equations $z_{1}=s_{1}(u, v), \ldots, z_{m+n-1}=s_{m+n-1}(u, v)$ as a graph over $D$.

Notice that the domain $D$ is foliated by the real-analytic arcs $\gamma_{u_{0}}$ : $b \Delta \cap \Delta_{1} \ni \zeta \mapsto c(\zeta-1)-b+u_{0} \in D$.

We write them as $[-d, d] \ni \theta \mapsto\left(u_{u_{0}}(\theta), v_{u_{0}}(\theta)\right)$, with $\zeta=e^{i \theta}$ and $c / 5=$ $\left|e^{i d}-1\right|$. Notice that by the disposition of $M_{1}$, if $\pi_{w}: \mathbb{C}^{m+n} \rightarrow \mathbb{C}_{w}$ denotes the projection, then $\pi_{w}\left(M_{1}^{-}\right) \cap \gamma_{u_{0}}([-d, d])=\emptyset$ for $2 b \leq u_{0} \leq 5 b$. Hence $\Sigma_{a, h}$ is foliated by the analytic arcs $\Gamma_{u_{0}}: \theta \mapsto\left(\gamma_{u_{0}}(\theta), s_{1}\left(u_{u_{0}}(\theta), v_{u_{0}}(\theta)\right), \ldots\right.$ $\left.\ldots, s_{m+n-1}\left(u_{u_{0}}(\theta), v_{u_{0}}(\theta)\right)\right)$ and for $u_{0} \geq 2$, these arcs are far from $M_{1}^{-}$.

Now, we complexify $\theta$ to a complex variable $\Theta \in[-d, d]+i[-5 b, 5 b]=: T$ such that $\operatorname{Re} \Theta=\theta$. Since $u_{u_{0}}, v_{v_{0}}$ and the $s_{j}$ are analytic, such a complexification exists and $\gamma_{u_{0}}(\Theta)$ yields a biholomorphism from this strip $T$ to a strip neighborhood of $\gamma_{u_{0}}([-d, d])$ in $D$ which contains, say, $\left\{\gamma_{u_{0}}(\theta)+u\right.$ : $\theta \in[-d, d],-3 b \leq u \leq 3 b\}=: D_{u_{0}}$. The domains of definition of the com- 
plexification are uniform since the piece $\Sigma_{a, h}$ comes from a real-analytic foliation, which can be supposed to be given by converging series in a fixed neighborhood of 0 . By the implicit function theorem over $D_{u_{0}}$, we can replace $\Gamma_{u_{0}}(\Theta)$ by a parameterizing variable $w=\Gamma_{u_{0}}(\Theta)$ so that $\Gamma_{u_{0}}(\stackrel{\circ}{T})$ will be a complex manifold of dimension one given by a graph $D_{u_{0}} \ni w \mapsto$ $\left(w, \varphi_{1}(w), \ldots, \varphi_{m+n-1}(w)\right) \in \mathbb{C}^{m+n}$ and which we will denote by $E_{u_{0}}$. Notice that $E_{u_{0}} \Subset \mathcal{U}$. Therefore, for all $u_{0}$ with $0 \leq u_{0}<5 b, E_{u_{0}}$ intersects $M_{1}$ transversally and $\Gamma_{u_{0}}([-d, d]) \cap M_{1}^{-} \subset \Gamma_{u_{0}}([-5 b, 5 b])$.

Now, inside the complex curve $E_{u_{0}}$, we can close up the analytic arc $\Gamma_{u_{0}}([-d, d])$ outside $M_{1}^{-}$, making the boundary of an analytic disc $B_{u_{0}}$ parameterized by $u_{0}$, a part of its boundary being given by $\gamma_{u_{0}}([-d, d])$ and the other part living in $M_{1}^{+} \backslash M_{1}$.

Notice that, by construction, all the $B_{u_{0}}$ for $0 \leq u_{0} \leq 2 b$ are analytically isotopic to each other and that all their boundaries are contained in $\mathcal{U} \backslash \mathcal{E}$. But $\Gamma_{0}(D)$ is a piece of the disc $B_{a, h}(\Delta)$ (recall that $\gamma_{B}$ is contained in $\Sigma_{a, h}$ ), so that $B_{0}$ is analytically isotopic to $B_{a, h}$ in $\mathcal{U} \backslash \mathcal{E}$ (isotope their boundaries inside $\left.a+h\left(L_{0}\right) \backslash M_{1}^{-}\right)$. And $B_{2 b}$ is analytically isotopic to a point in $M_{1}^{+} \backslash M_{1}$. This yields the desired isotopy.

The proofs of Theorems 5.10 and 5.1 are complete.

\section{References}

[1] E. Chirka, Complex Analytic Sets, Kluwer, Dordrecht, 1989.

[2] E. M. Chirka and E. L. Stout, Removable singularities in the boundary, in: Contributions to Complex Analysis and Analytic Geometry, Aspects of Math. E26, Vieweg, 1994, 43-104.

[3] T.-C. Dinh and F. Sarkis, Wedge removability of metrically thin sets and application to the CR meromorphic extension, preprint, 1997.

[4] P. Dolbeault et G. M. Henkin, Chaînes holomorphes de bord donné dans $\mathbb{C P}^{n}$, Bull. Soc. Math. France 125 (1997), 383-446.

[5] F. R. Harvey and H. B. Lawson, On boundaries of complex analytic varieties, Ann. of Math., I: 102 (1975), 233-290; II: 106 (1977), 213-238.

[6] S. M. Ivashkovich, The Hartogs-type extension theorem for meromorphic maps into compact Kähler manifolds, Invent. Math. 109 (1992), 47-54.

[7] B. Jöricke, Removable singularities for CR-functions, Ark. Mat. 26 (1988), 117143.

[8] -, Envelopes of holomorphy and CR-invariant subsets of CR-manifolds, C. R. Acad. Sci. Paris Sér. I 315 (1992), 407-411.

[9] -, Deformation of CR-manifolds, minimal points and CR-manifolds with the microlocal analytic extension property, J. Geom. Anal. 6 (1996), 555-611.

[10] - Some remarks concerning holomorphically convex hulls and envelope of holomorphy, Math. Z. 218 (1995), 143-157.

[11] - Boundaries of singularity sets, removable singularities, and CR-invariant subsets of CR-manifolds, preprint, 1996. 
[12] G. Lupacciolu, A theorem on holomorphic extension of CR-functions, Pacific J. Math. 124 (1986), 177-191.

[13] C. Laurent-Thiébaut, Sur l'extension de fonctions $C R$ dans une variété de Stein, Ann. Mat. Pura Appl. (4) 150 (1988), 141-151.

[14] J. Merker, Global minimality of generic manifolds and holomorphic extendibility of CR functions, Internat. Math. Res. Notices 8 (1994), 329-342.

[15] - On removable singularities for CR functions in higher codimension, ibid. 1 (1997), 21-56.

[16] J. Merker and E. Porten, On removable singularities for integrable CR functions, preprint, 1997; available at: http://www.dmi.ens.fr/EDITION/preprints.

[17] E. Porten, thesis, Berlin, 1996.

[18] -, A Hartogs-Bochner type theorem for continuous CR mappings, manuscript, 1997.

[19] F. Sarkis, CR meromorphic extension and the non embedding of the AndreottiRossi CR structure in the projective space, Internat. J. Math., to appear.

[20] B. Shiffman, Separately meromorphic mappings into Kähler manifolds, in: Contributions to Complex Analysis and Analytic Geometry, Aspects of Math. E26, Vieweg, 1994, 243-250.

[21] J.-M. Trépreau, Sur la propagation des singularités dans les variétés CR, Bull. Soc. Math. France 118 (1990), 403-450.

[22] A. E. Tumanov, Connections and propagation of analyticity for CR functions, Duke Math. J. 73 (1994), 1-24.

Laboratoire d'Analyse, Topologie et Probabilités UMR 6632

Centre de Mathématiques et d'Informatique 39 rue Joliot Curie

13453 Marseille Cedex 13, France

E-mail: merker@gyptis.univ-mrs.fr
Max-Planck-Gesellschaft Humboldt-Universität zu Berlin Jägerstrasse, 10-11

D-10117 Berlin, Germany E-mail: egmont@mathematik.hu-berlin.de 